\title{
Mind the Gap: A Seven-Question Field-Map Highlights Consciousness Science's Critical, Neglected, Terrain
}

\author{
Nicholas M. Rosseinsky ${ }^{1}$ \\ ${ }^{1}$ Consciousness and Computational Neuro/Physics Lab, Departments of Neuroscience and Physics, \\ Center for Dialog in Science, London, U.K. \\ * Correspondence: rosseinsky.nicholas.m@cfdis.org \\ Keywords: consciousness, phenomenal consciousness, hard problem of consciousness, \\ integrated information theory, global neuronal workspace, higher-order thought, predictive \\ coding, Orch-OR.
}

\begin{abstract}
As a way of mapping complicated theory-landscapes in consciousness science, I identify seven questions applicable to individual theoretical proposals. This seven-question inventory comprehensively probes currently-crucial attributes for serious scientific theories-of-consciousness.

I then illustratively apply the question-inventory to five leading theories-of-consciousness (IIT, GNW, PC, HoT and Orch-OR), highlighting similarities and differences. Under a simple quantification system, the median score is $1.5 / 7$ (or about $20 \%$ !), and the field-leader is Orch-OR (but still scoring only $2.5 / 7$ !). Beyond these introductory illustrations, the seven-question approach can map all theoretical stances (including Dennett's sometimes difficult-to-understand position), and identify what's critically missing from today's field.
\end{abstract}

\section{$1 \quad$ Introduction}

Consciousness science can be a confusing field. (One rather fundamental confusion seems to be about whether and when the field can even exist, as a 'science'!)

On the one hand, science is apparently solving problems that stumped philosophers for centuries (Koch, 2018). We're bound to finish up this scientific explanation of consciousness (Block et al., 2014). Slightly-less definitive stances are nevertheless similarly positive (Seth, 2018a; Storm et al., 2017). It's just a matter of choosing between options like integrated information theory (Tononi et al., 2016), global-neuronal-workspace theory (Baars, 1988; Dehaene and Changeux, 2011), predictive coding (Hohwy, 2013) and higher-order thought (Lau and Rosenthal, 2011) (all of which explain consciousness in terms of more-or-less conventional, classical, matter). We have reliable experiments that we can use to make evidence-based choices between those theoretical options (Koch et al., 2016). Although there's disagreement about what inferences to make from existing experimental-data (Boly et al., 2017; Odegaard et al., 2017), again there's allegedly no doubt that consensus will be achieved (Block et al., 2014).

[Note that even within this first, apparently-single, grouping - which I call mainstream consciousness science - there's a deep split between those who believe we can, should, and do study conscious experience or phenomenal consciousness (Block, 2005), and those who focus on access consciousness, which itself means different things to different people, In at least one version, 'access' simply means 
'what we are able to report on', and there are then debates about the degree how this relates to what we consciously experience, in the phenomenal sense (Phillips, 2018).]

On another, quantum, hand, there are allegedly things that human brains do that classical matter can't (Lucas, 1961; Penrose, 1989). That capacity (amongst other things!) is the basis for a necessarilyquantum approach to conscious experience [Orchestrated Objective-Reduction, or 'Orch-OR' (Hameroff and Penrose, 2014)]. Again, there are experiments (Sahu et al., 2013)!

On third, fourth, and fifth physical/philosophical, hands: conscious experience is impossible to examine scientifically, under standard physics (Cohen and Dennett, 2011; Elitzur, 1989; Hawking, 2000; Rosseinsky, 2018a; Wilkes, 1988); and/or, there's a 'hard problem' (Chalmers, 1996) that noone has made any progress on (Goff, 2017), and even a 'meta-problem' (Chalmers, 2018) that seems even more intractable (if that's possible!) ; or, the whole thing is really unclear (Overgaard, 2017) but we should definitely be extremely careful, humble and tentative, both when investigating experience, and in making claims about current theories of it (Bayne and Spener, 2010; Paller and Suzuki, 2014; Phillips, 2018).

On a sixth, sociological, hand (Smolin, 2007), there are three major conferences every year with the words 'science' and 'consciousness' (or the equivalent!) in their titles, each with a distinctive worldview (or one might even say dogma!), and very little cross-talk between the three. (They are: 'The Science of Consciousness'; the annual meeting of the Association for the Scientific Study of Consciousness; and, 'Science and Non-Duality'.) This is quite unlike any other scientific discipline, where distinct $s u b$-field conferences often occur, but mutually-contradictory field-faction meetings never persist.

On a seventh, explain-it-to-me-like-I'm-a-five-year-old, hand: what is going on in consciousness science? Of course, it's OK for there to be many views, and for people to disagree. But if this field really is 'science', surely the theoretical and empirical grounds for disagreement should be clearly on display - and claims of definitive progress should be moderated by naming serious, dissenting, views.

Or should they? For example, in debates about climate change, one view is that news organizations give too much room to dissenters, potentially creating doubt in the minds of laypeople about where the balance of scientific analysis lies. Maybe the situation in consciousness science is like that. Maybe mainstream consciousness science has it all right, and is ignoring the other views because they're wrong and dangerous.

My own view is quite the opposite: the danger presently is in the mainstream's failure to really look at alternative viewpoints, and to appreciate serious deficiencies in all of its proposals. (Not that alternatives to the mainstream are totally deficiency-free - far from it, as we'll see!) Worryingly, the very same mainstream has significant influence in many of the leading journals in the field, leading to the potential for a disturbing groupthink. Students and young investigators follow the tone set in the literature - and that tone is growing in its unquestioned acclaim of the mainstream (Dehaene et al., 2017; Koch, 2018).

Of course, my personal view isn't important. What matters is the truth-of-the-matter! In this paper, I construct a seven-question inventory (Table 1) for theories-of-consciousness, with the intention of facilitating a collective enquiry into the truth about the current field. I have no skin-in-the-game: I'm not advancing a specific theory. Nor am I trying to tear down anyone, or anything: my interests are solely constructive - I want to see a coherent scientific approach to consciousness (and I judge that we don't have that, in the current fractious, fragmented, irrational, process!). 
Table 1. The Seven Questions. For more detail on Q1 to Q7, please see Sections 3 to 9. (Q1 and Q2 are introduced in Section1.) Section 2 discusses why these seven specific questions have been chosen at this point, over other candidates. It also briefly reviews various applications of the questioninventory, and potential problems of subjectivity in formulation, and evaluation. (In each question, 'theory- $X$ ' is a placeholder for the name of the specific theory being investigated.)

\begin{tabular}{|c|c|}
\hline \# & Question-Content \\
\hline Q1 & Object-of-study. 'Does theory- $X$ study and offer explanations for conscious experience?' \\
\hline Q2 & $\begin{array}{l}\text { Causal Efficacy. 'Does theory- } X \text { assume that contents-of-conscious-experience have causal } \\
\text { efficacy, in the sense that there can be transfer-of-information about the complete contents of } \\
\text { experience, into brain-activity?' }\end{array}$ \\
\hline Q3 & $\begin{array}{l}\text { Evolutionary Advantage. 'Does theory- } X \text { provide a direct evolutionary advantage for } \\
\text { experience?' }\end{array}$ \\
\hline Q4 & $\begin{array}{l}\text { Detailed Cortical-Relationships. 'Does theory- } X \text { state or create detailed, explicit. relationships } \\
\text { between components-of-experience, and cortical-activity that encodes specific aspects of } \\
\text { body/environment, and/or governs specific behavioral/somatic activities?' }\end{array}$ \\
\hline Q5 & $\begin{array}{l}\text { Locality/Geometries. 'Does theory- } X \text { offer an explicit solution to the tension between } \\
\text { orthodox-physics locality, and the difference between brain-encoding geometry and } \\
\text { experiential geometry?' }\end{array}$ \\
\hline Q6 & $\begin{array}{l}\text { Lawfulness/Individuated-Multiplicity. 'Does theory- } X \text { account for the distinct, individuated, } \\
\text { experience-streams of multiple-experiencers - especially if it proposes the same encoding-to- } \\
\text { experience associative-laws apply to each experiencer?' }\end{array}$ \\
\hline Q7 & Special-Relativistic Consistency. 'Is theory- $X$ special-relativistically-consistent?' \\
\hline
\end{tabular}

Naturally, the seven questions - and the ways I use them to evaluate theoretical proposals - implement a point-of-view. But my intention is to ground that point-of-view's comparative, evaluative, aspects solely in two priors. First, science! What does it mean for an enquiry into consciousness to be 'scientific'? (Naturally, defining 'science' can again involve subjective choices. However, I aim to be transparent. And some aspects should be uncontroversial, e.g. 'science must have reliable experiments'.) Second, the very nature of the phenomenon we are trying to study. What is 'conscious experience' - and can it even be meaningful to talk about it?

The emphasis on conscious experience (or phenomenal consciousness) - if we can study it without introducing contradictions and inconsistencies - is my first subjective choice:

A1: Object-of-study. Consciousness science should centrally include the study of conscious experience, if it is possible to do so in a contradiction-free, scientifically-rigorous, way.

(A-labelled statements are assumptions, or axiomatic-choices, made in establishing this paper's evaluative structure.) I try to use the word 'consciousness' as little as possible, but when I do, it means 
'conscious experience, possibly plus some other things' - i.e. consciousness is an open-boundary collection of phenomena that minimally includes experience (again, if we can even speak about that!). Why emphasize conscious experience, over, for example, access consciousness? It just seems to me that this is the crucial feature-of-Nature that demands explanation. Of course, it may not be possible to study it scientifically. But let's see what the issues are, and under what conditions we could study it. If we can't, then let's study what we can - and be clear we are not studying experience. Choosing to orient the field towards conscious experience means that the first of the seven questions probes exactly that issue (Q1, Table 1).

Why do I repeatedly refer to 'even being able to speak about conscious experience, or define it?'. Isn't it obvious we can speak about experience? Well, it may be 'obvious' - but many things that appear obvious aren't true, and one of the roles of science (my first ground) is to reveal truth! The issue here is whether we can talk scientifically about experience, if experience doesn't couple with (influence, affect, transfer information to) brain-dynamics (Q2, Table 1). (Trivially, causal-efficacy/dynamicalcoupling does not require dualism of any kind.)

In some ways, this is simple and straightforward. If conscious experience doesn't influence the brain's matter-dynamics, which govern and generate reports-of-all-kinds, it seems obvious that there can't be any experiments about detailed contents-of-experience (Cohen and Dennett, 2011), and there can't even be a meaningful definition of experience-as-a-whole (Chalmers, 2018). Then again, how are we to explain everyday intuitions that report is always about experience - and the vast and growing allegedly-scientific literature about experience (Block et al., 2014; Fahrenfort and Lamme, 2012; Koch et al., 2016; Storm et al., 2017)? Suffice to say this apparently simple issue is in fact quite complex! Based on extensive treatment elsewhere (Rosseinsky, 2018a), my second subjective choice is to assert fatal problems for a science of conscious experience, without casual-efficacy:

A2: Causal efficacy. If conscious experience doesn't dynamically-couple with brain-dynamics (or, synonymously, 'isn't causally-efficacious with respect to brain-dynamics') in specific ways, there are no scientifically-meaningful experiments about experience, and it's not even scientifically-meaningful to refer to 'conscious experience' (e.g. as I did at the start of this assumption!).

Likely, many of those who favor an access-consciousness field-approach stopped reading at A1. Many of those who think we can study conscious experience without causal-efficacy probably stopped at A2. One thing this indicates is that Q1 and Q2 (and related A1/A2-assumptions) are effective in mapping the field! (I'm not reasoning from people-stopping-reading to effectiveness! I mean that Q1 and Q2 are mapping-effective because they already parcel off large and significant field-divisions: access vs phenomenal consciousness in Q1, and then phenomenal consciousness with/without causal-efficacy, in Q2.)

For those who interested in pursuing this Question/Axiom approach to mapping the field, Sections 3 and 4 amplify Q1/Q2-introductions given so far, and Sections 5 to 9 expand on Q3 through Q7 (Table 1). Section 10 then uses Q1-to-Q7 to examine five major current theories [integrated information theory (Tononi et al., 2016), global neuronal workspace (Baars, 1988; Dehaene and Changeux, 2011), predictive coding (Hohwy, 2013), higher-order thought (Lau and Rosenthal, 2011), and Orch-OR (Hameroff and Penrose, 2014)]. Section 11 discusses the resulting observation that these 'Big Five' all neglect some important theoretical terrain - and examines how that terrain could be occupied, in future. Section 12 summarizes the implications of earlier Sections for the field as a whole, and closes with this 
question: can the seven-question approach catalyze a constructive, communal, enquiry into what's happening in the field?

\section{Using Seven Questions to Map and Evaluate the Field}

Before launching into the journey that begins in Section 3, this Section steps back, to examine the content and structure of the seven-question approach, as a whole. Why these seven questions, and not others? Where exactly have I inserted subjective preferences and assumptions? How can we to use questions to evaluate theories? (Should we?!) Even assuming that we can establish objective, uncontroversial answers to questions like 'Does theory-X establish detailed relationships between specific brain-activity and specific contents-of-consciousness?' (Q4) ... is 'yes' a better answer than 'no'? Why?

\subsection{Basic Frame: Experience, an Unseen 'World', and Science}

\subsubsection{Indirect/Representational Realism: Experiential and Unseen Realms}

This Section steps back, to evaluate the seven-question approach itself. But what frame do we step back into? Without declaring that frame and contrasting it with alternatives, I risk injecting unexamined subjective content, or creating a dialogue that starts with crossed-lines.

I adopt the frame of indirect or representational realism (Lehar, 2000), as a starting point (Fig. 1). All sights (including those of my own body), all sounds (including 'the voice in the head' ), all sensations (tactile, proprioceptive, emotional) can be variously described as: aspects of my mind; features of consciousness; a virtual re-presentation of an unseen realm-of-reality (Kant et al., 1998) in my phenomenal- or experiential-space (Smythies, 2012); and so on:

A3: Indirect-realism/representationalism. The 'indirect realist' or 'representationalist' views are accurate, in the sense that objects in human conscious experience aren't directly the matterobjects that physical theory discusses, but indirect versions that re-present those matter-objects, within a personal virtual-reality.

Conventional 'hard' sciences (i.e. physics, chemistry, biology) all examine and explain an apparent dynamical order in the unseen reality-realm (Fig. 1; 'realm' doesn't imply dualism!). Consciousness science is then an attempt to explain (if possible!) the instantiation and movement of experience (sights, sounds, sensations) relative to, and in terms of, the presumed existence and movement of the unseen realm. (This may seem like a declaration that consciousness science is focused only on phenomenal consciousness, but that's not so, e.g. because of the reference to movement: see subsection 2.4.2.1.)

\subsection{2 (Optional) Symbol-based Expression of Consciousness Science}

Because all the symbols in conventional science (' $e^{-}$' for 'electron', $\mathbf{r}$ for location, etc.) refer to the unseen realm, it can be useful to introduce a symbol $\langle s\rangle$ to label experience-of an unseen-realm stimulus labelled $s$ [(Rosseinsky, 2018b); Fig. 2]. This approach is pursued in the Supplementary Material, which offers a symbol-based analysis paralleling that of the main-text.

\subsubsection{Multiple Human-Experiencers}

Finally, if there are multiple human-experiencers observing a shared scene (Fig. 3A), there is a common unseen-reality and multiple, separate, individuated, experiencing-streams. Contrast typical consensual 
Figure 1. Experiential and 'Unseen' Realities. A. Two-realm depiction of reality. Semi-transparent grey rectangle schematically separates 'unseen reality' from 'conscious experience'. 'Conscious experience' (D1) includes thoughts (and imaginations), audiovisual experience of the unseen environment (depicted here schematically by a colored photo representing visual experience, plus the placeholder text 'Sounds'), and feelings of all kinds (emotional, tactile, and proprioceptive). 'Unseen reality' contains all the objects studied by conventional science (i.e. science excluding the study of conscious experience!). Purple rectangles schematically depict the entire physical Universe. Two unseen-realm objects are schematically-emphasized: the physical tree (purple line-drawing) that is represented centrally in the experiencer's visual experience, and the physical brain (purple line-drawing) of the experiencer (who is experiencing thoughts, sounds, feelings, and colored visual-experience of the tree). B. We have no knowledge of the 'actual constitution' of 'unseen reality'. Exactly-the-same conscious-experiences are completely-consistent with an alternative view, in which 'objects' in the unseen realm exist as numbers ('\#'-symbols), which are recomputed according the laws of physics. (For example, some subset of the numbers depicted corresponds to the physical state of the experiencer's brain. As well as the capacity to recompute unseen-realm numeric-content, Nature contains the capacity to 'project' specific experiential-content, according to the numeric-state of the experiencer's brain). Note that 'two realms' doesn't imply dualism, but simply reflects logical features of reality. All conventional science concerns inter-relationships between purple entities in a single realm; conscious-experiential science concerns inter-relationships between realms.
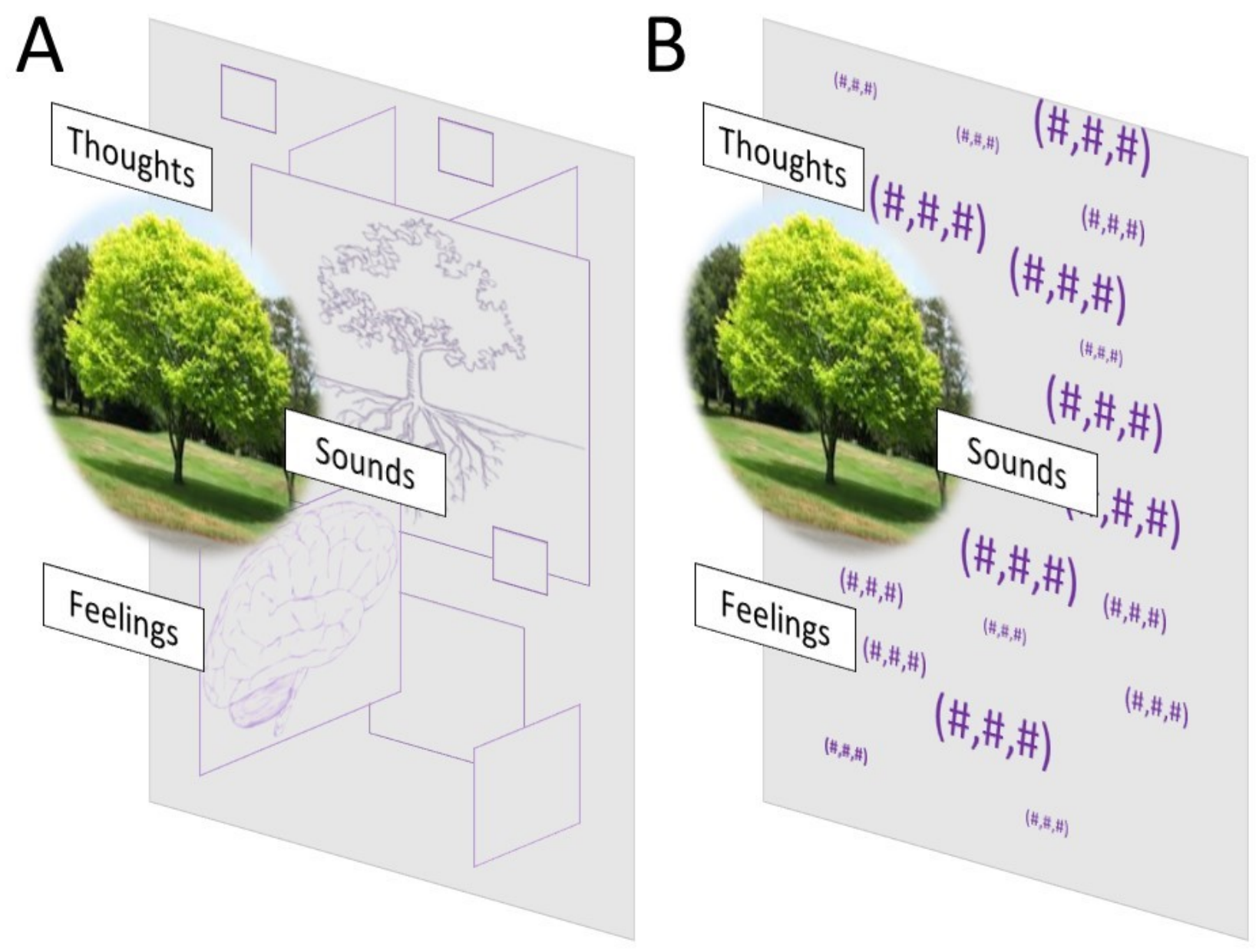
Figure 2. Symbols for Conscious Experience. A. A matter-tree (schematically-represented by the purple line-drawing) and the corresponding experienced-tree (color-photo) aren't the same (subsection 2.1, main-text; Fig. 1). Existing symbols in physical theory, such as ' $e^{-}$' (electron) and 'r' (spatiallocation), properly label (blue double-headed arrows) aspects of the unseen-realm (Fig. 1), only (above the dotted line). The question-mark indicates absence of equivalent symbols for conscious experience (below the dotted line). B. Symbols $\left\{s_{\mathrm{i}}\left(\mathbf{r}_{j}\right)\right\}$ are introduced to label macroscopic, brain-encodingrelevant, features of the matter-realm. (' $\subset$ ' indicates these symbols belong to the overall class of " $\left\{e^{-}\right.$, r\}-like" symbols.) A novel symbol $\left\langle s_{\mathrm{i}}\right\rangle\left(\boldsymbol{\rho}_{j}\right)$ then labels the component of conscious experience that corresponds directly to the $s_{\mathrm{i}}\left(\mathbf{r}_{j}\right)$ stimulus (macro-aspect of the unseen-realm). For example, the two visually-experienced tree-edges (colored contents of red squares, lower-half) correspond to respective matter-edges (purple lines in red squares, upper half). Note the introduction of a new spatial-coordinate symbol $\boldsymbol{\rho}$. Sections 6 to 9 (Q4 to Q7) examine aspects of the $\mathbf{r} / \mathbf{\rho}$ relationship, and of the relationship between the spaces they index. C. Another set of " $\left\{e^{-}, \mathbf{r}\right\}$-like" symbols, $\{\mathbf{A}(\mathbf{r})\}$, label generic brainactivity (red spikes) and configuration, at a set of brain-loci $\mathbf{r} \in \mathbf{r}_{\mathrm{B}}$ (schematically depicted by the redellipse, left-hand side). A detailed theory-of-conscious-experience must include a function $f$ relating unseen-realm symbols (contents of the black-ellipse, right-hand side), describing stimuli and brainstate, to new conscious-experiential symbols $\left\{\left\langle s_{\mathrm{i}}\right\rangle\left(\boldsymbol{\rho}_{j}\right)\right\}$. See Supplementary Material for a more detailed account of this symbol-based approach, and its application to mapping and evaluating theories.

\section{A Phenomena Symbols C Phenomena Symbols}

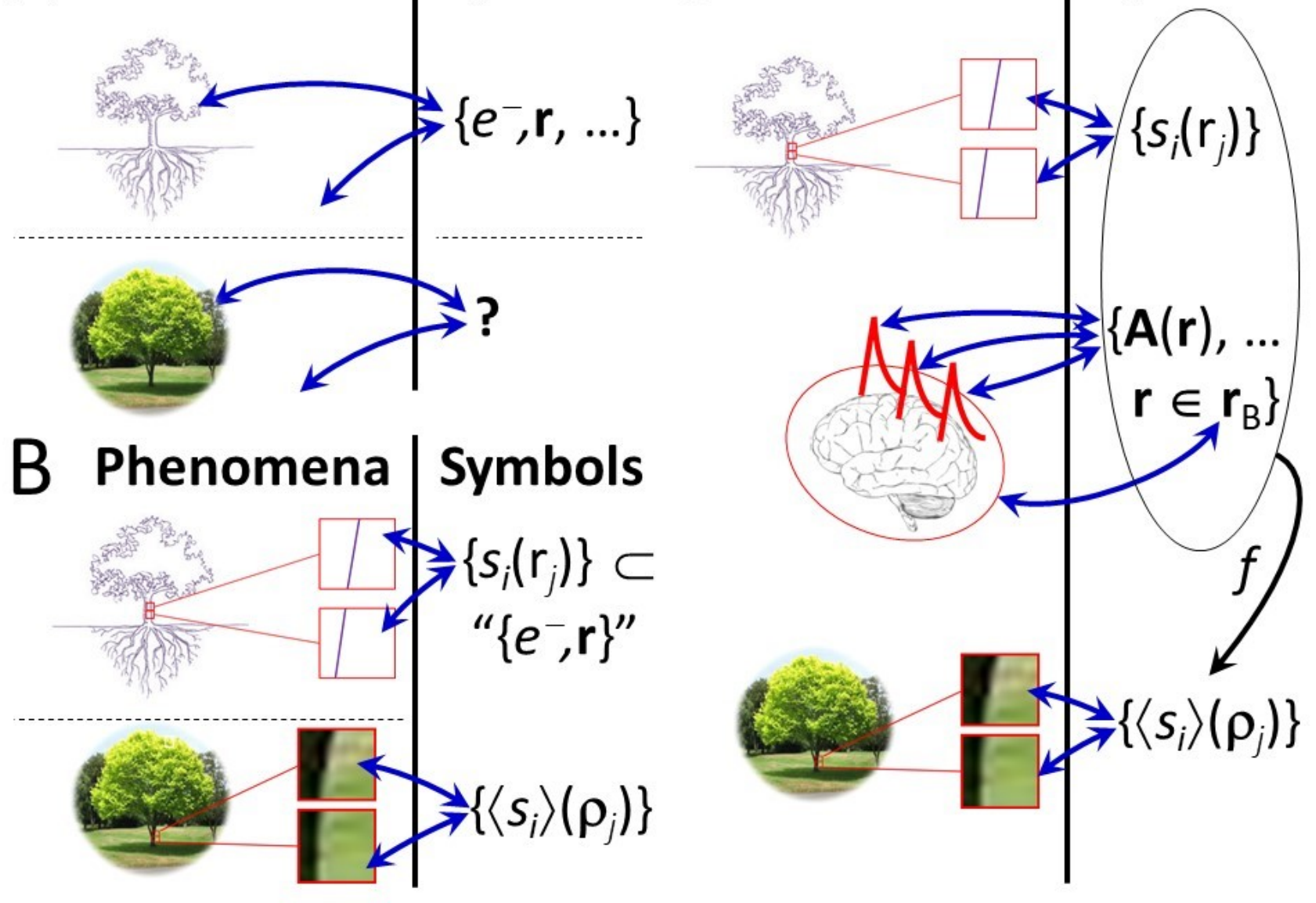


Figure 3. Multiple Experiencers. A. Multiple experience-stream view. (This panel reproduces panel A of Figure 1, but with the addition of a second brain/experiencer.) One brain (schematically depicted by a red-bordered purple-line drawing) observes a scene including an unseen-realm tree (purple-line drawing), and associates with experiences of thoughts, feelings, sounds, and a visual image of the tree (red text, red-bordered circle). Another brain (blue-bordered purple-line drawing), observing the same scene from a different angle, associates with its own thoughts and feelings (dashed-text, blue-borders), and its own audiovisual experiencing-stream (blue-bordered circle, 'Sounds', correlates of auditory experience are the same for both brains, the experience-stream will be different because it is created relative to a different point-of-view - compare the visual experience, where is it obvious that a different point-of-view creates a different experience!). B. Conventional/consensual view. A typical, everyday, interpretation of two experiencers in a common scene: the audiovisual experience is shared objective reality, and only thoughts and feelings are private. This everyday view can't explain simple illusions (eye-crossing makes objective reality split in two!), and doesn't align with basic science (e.g. the role that unseen-realm dynamics play in creating brain-conditions for visual experience, emphasizing that there $i$ s some form of unseen realm - which is completely excluded by the naïve realist view that audiovisual reality is that unseen realm!). This paper assumes that multiexperiencer reality is better depicted by panel A, than panel B.

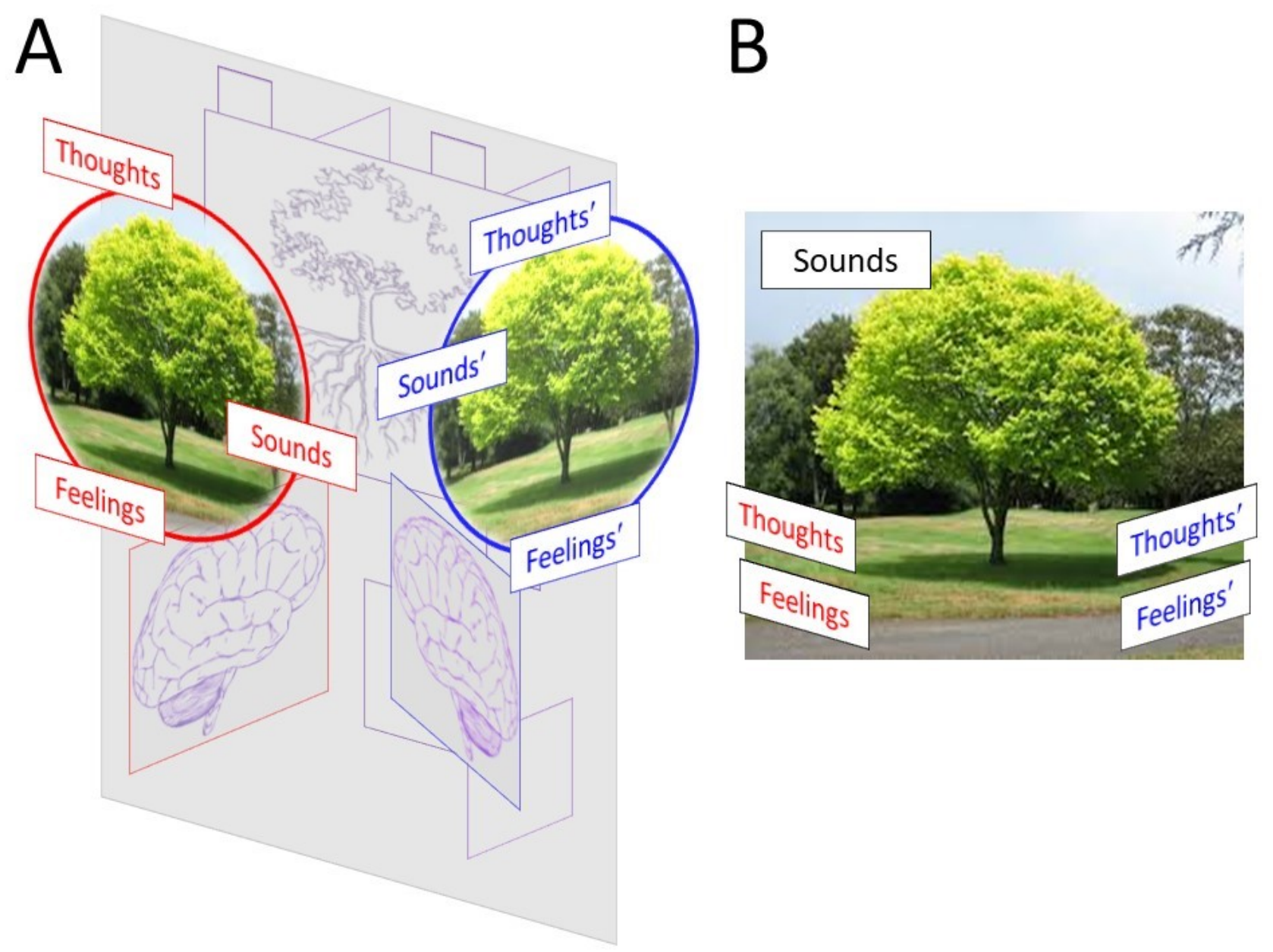


interpretations, in which 'my experience' is reality-itself, and another experiencer is just viewing that reality from another angle (Fig. 3B).

\subsubsection{Alternative Frames}

The primary alternative frame that seems to creep in repeatedly - sometimes even when commentators have declared that they discarded it! - is that experience is the same thing as unseen-reality (equivalently, there's only one reality-realm). For example, 'what it's like' definitions of conscious experience (Nagel, 1974) tend to imply that experience is limited to thoughts and feelings, and neglect the fact that the entire audiovisual 'environment' is also part-of-experience. Another common alternative, in the multi-experiencer context, is to neglect multiple experiencing-streams (Fig. 3B). Slipping into these illogical alternatives matters: they can make subsets of Q4 to Q7 (Table 1) appear meaningless, and can thus make theories-of-experience look coherent, when they are not.

\subsection{Preview: Subsections 2.3 to 2.5}

Relative to this basic frame, I now examine the general question of alternate question-lists (subsection 2.3), alternatives to specific questions in the current list-version (subsection 2.4), and different ways of applying the current question-list (subsection 12).

\subsection{Alternative Inventory-Versions}

\subsubsection{Alternative Versions, Now: More-Detailed Answers}

Subsection 2.4 will defend specific questions chosen in Table 1. In a structured use of the questioninventory, we also need to pre-define possible answers to the questions. For example, subsection 10.1 defines a simple, specific, answer-set, which Section 10 then applies to 'the Big Five'.

Simple answer-sets used in this introductory paper could in principle be developed immediately into more detailed and refined versions, which would parse the field in a more nuanced way. For example, subsection 10.1 uses a relatively-crude 'yes/sometimes-partially/no' answer-structure for Q1 and Q2. Figures 4 and 5 illustrate the different mapping capabilities arising from a fundamentally-different answer-structure (for just these two questions), using four detailed-categories for each question.

\subsubsection{Alternative Versions, In the Future: Different Questions}

In ten years - or even two! - the question-list should be completely different. That is, the seven questions here aren't meant to capture 'timeless issues for the field'. They are the most significant issues now, and therefore the best way to understand relative merits of presently-competing theories.

\subsection{Why These Seven-Questions?}

This subsection discusses why the seven questions don't specifically address other definitions of consciousness (subsection 2.4.1), philosophical/metaphysical controversies (subsection 2.4.2), or detailed-assessment of theories against experimental data (subsection 2.4.3).

\subsubsection{Why Aren't There Questions About Other Conceptions-of-Consciousness?}

Q1 and A1 seem to put the focus on conscious experience (phenomenal consciousness). What about access-consciousness, and/or level-of-consciousness (or 'state')? 


\subsubsection{Why Isn't 'Explanation of Serial Information Processing' Included?}

Q1's focus on conscious experience might seem to unfairly de-prioritize work on access consciousness. However, subsection 2.5 points out the difference between mapping and evaluating: asking-about conscious experience doesn't inherently judge theories not-about-experience to be of lesser value. And Table 2's answer structure that explicitly mentions access-consciousness in three-out-of-four answercategories.

And, to be transparent, A1 does consider explanation-of-experience (if possible!) to be a greater and more important scientific mystery, than 'merely' explaining how the brain's massively-parallel and distributed information-processing also gives rise to a singular stream of serial information-processing and decision-making (which is one version of the access-consciousness project).

\subsubsection{What About 'Explains Content' vs 'Explains State/Level' Distinctions?}

Certainly, some theories provide better explanations of levels and states of consciousness than others. A future question-inventory (subsection 2.3.2) could use a question about level-theoretic-capacity, to distinguish between theories. But, in my judgement, a far more pressing field-issue currently is the A2disconnect between talking about experience, but not proposing causal-efficacy. Without fixing that, better-theorizing-about-levels is just a more sophisticated form-of-contradiction!

\subsubsection{Why Aren't There Questions About Philosophical/Metaphysical Controversies?}

One objection to all 'consciousness science' is 'it doesn't address "the hard problem"'. Another slew of objections concerns things like inverted qualia and 'other minds'. Shouldn't questions about these kinds of issues be on the list?

\subsubsection{What About the 'Hard Problem'?}

The 'hard problem' is, allegedly, that there is no reason why the brain should associate with experience, and/or no explanation for how it creates or participates in such associations. Scientifically, however, the 'hardness'-attribution is meaningless. Consider the 'hard problem of electrons': 'There's no explanation for why or how electrons have electric charge'. 'The hard problem of spacetime curvature': 'There's no explanation for how or why mass-energy causes spacetime curvature'. And so on. And on. And on.

The hard problem - if it's taken to be a scientific issue - is just a mistake about the nature of science (Mukhopadhyay, 2018):

A4: Basic quality of science. Science is the identification of orderly structural and dynamic relationships between various natural phenomena.

Here's an exemplar scientific-solution of 'the hard problem': 'One natural phenomenon is conscious experience. Another natural phenomenon is a human brain. When brains are in these states, they associate with these conscious experiences'. This is precisely 'the identification of an orderlystructural/dynamic relationship', just as electric charge is a key part of the orderly-structural/dynamic relationships between electrons and other fundamental particles.

Of course, we want to know precisely what it is about brains that leads to such associations, so that we can look at other systems and say 'yes we predict consciousness here', or 'this empirical marker demonstrates there is consciousness there'. There are various, difficult, scientific problems associated with project. One is, if experience isn't causally-efficacious, we can't even talk about it or define it, let 
alone do experiments on it. That makes it impossible to study consciousness, not hard! Another problem - e.g. for the exemplar-solution suggested above - is that it tends to make brains appear in fundamental law, which violates current structural features of science. And, of course, it doesn't enable comparative analysis with other non-brain systems. But let's just face up to these scientific issues for what they are, rather than creating illusory ones by misinterpreting the basic quality of science!

\subsubsection{What About Other, Standard, Philosophical Problems?}

It's sometimes suggested that various issues like inverted qualia (Shoemaker, 1982), the problem of other minds (Locke, 1998), or psychophysical parallelism (Wegener, 2009) could derail consciousness science. I don't include a question about these issues, because I believe they can all be satisfactorily addressed (for the time being!) by suitably-nuanced, tentative, assumptions (provided these assumptions are kept clearly in sight, and regularly reviewed). For example, it's been suggested that the 'problem of other minds' can be addressed via an abductive assumption.

\subsubsection{What About Ontology?}

I take the view that science is ontology-free. That may seem like an unusual position, because science is sometimes seen as explanation of natural phenomena basically in terms of matter, in which case science seems to be inherently a monist materialist enterprise. But in A3's indirect/representationalistframe, we never see e.g. particles/fields themselves: they could 'exist as' numbers [Fig. 1B; computationalism (Wheeler, 1990), or ontic structural realism (Ladyman, 1998)], or as aspects of a Universal mind [a form of idealism (Berkeley, 1878)], or as matter (whatever that means!) - or all of the preceding ... or none of them! What matters (A4) is the orderly dynamic relationships between explanatory entities (particles/fields!), not 'what they're made of'!

\subsubsection{Why Aren't There Questions About Experiments - If This Is About Science?}

Whether or not we attribute inherent ontological positioning to science, one of science's defining qualities is certainly use-of-experiments. Surely scientific theories should be compared against experimental data, primarily - so why are there no inventory-questions directly about experiments?

\subsubsection{What About Consistency with (Past) Experimental Data?}

Naturally, relative consistency with existing experimental data is a critical means for evaluating competing theories. But if consciousness science is (or should try to be) minimally about conscious experience (A1), then experiments can only be scientifically-valid if theories propose causal-efficacy (A2). Conversely, in the absence of causal-efficacy, existing experimental data isn't about conscious experience (Rosseinsky, 2018a). So, the pressing issue, before comparing theories with data, is to establish the relevance of any experiential-data to conscious-experiential science!

\subsubsection{What About (Future) Experimental Testability?}

Hypotheses can be more useful scientific contributions when they're testable against existing proposals. Thus, it's true that a theory equipped with good experimental tests should score more highly on some future question-inventory than one that does not (all else equal). But the presently-critical experiment is one that tests whether causal-efficacy exists [Q2; (Rosseinsky, 2018a)]. [Although it might seem like experimental support for causal efficacy would count against no-efficacy mainstreamtheories, in fact most theories could be easily revised to cohere with such results (subsection 11.1.1).] 


\subsubsection{From 'Why Not Other Questions' To 'Why These'}

Subsections 2.4.1 to 2.4.3 explain why certain questions aren 't included. That still doesn't explain why Table 1's choices are selected, especially because Q5-to-Q7 (and perhaps Q2 and Q4) may seem unfamiliar, or even arbitrary. The basis for these choices is Section 1's first ground: science. Taking science to be an integrated knowledge-system, consciousness science must cohere with physics (or, catalyze evidence-based changes in physics, around mutual inconsistencies). Q2 and Q4-through-Q7 probe the physics-consistency of consciousness science.

\subsection{Using the Seven-Questions: Mapping or Evaluating?}

\subsubsection{Mapping}

To use Table 1's questions for mapping, we simply define answer-sets for each question, and then use answers-for-each-theory to locate that theory in a seven-dimensional space. Figure 4 demonstrates a two-dimensional depiction of a four-dimensional mapping-process for six theories-of-experience, using Table 2's answer-sets for Q1 and Q2 (subsections 3.2and 4.2), and Section 10's analyses for Q3 and Q4.

\subsubsection{Evaluating}

Mapping is a relatively-objective activity (although questions and answer-sets can enact subjective biases!). Evaluating - assigning a 'better/worse' judgement - is much trickier! Subsections 2.5.2.1 and 2.5.2.2 briefly discuss qualitative vs. quantitative evaluations, respectively.

\subsubsection{Complex Qualitative Assessment}

A map (or its underlying structured assessment-tool) can help facilitate complex qualitative assessments, e.g. by identifying areas of terrain that are inconsistent. For example, Figure 5 highlights the inconsistency of theories-about-experience without causal-efficacy (which we've already discussed at length), but also problems for theories-about-experience with the 'wrong sort' of causal-efficacy (Fig. 5: red shaded area, top-right).

\subsubsection{Simple Quantification}

By assigning numeric-scores to a structured answer-set, and defining a way to combine values across questions, it's possible to score individual theories, and even the field as a whole. The point of such an exercise is just to give another way of seeing what's going on. Certainly (subsection 10.4), reporting that the five best theories in the field score a median of $21 \%$ and a maximum of $36 \%$ is one way to focus attention. It's sometimes said: what gets measured gets managed!

\subsubsection{Subjective vs Objective Content in Questions/Assessment}

Even if everyone agreed that Table 1 contains the seven most significant questions for the present field, and even if everyone agreed on the actual answers, I'm not claiming that everyone would evaluate those answers in the same way! That is, as well as possibly-subjective content in question-choice and in formulating answer-lists, judging whether 'yes' or 'no' (say) is a 'better' answer can be a subjective and contentious matter (and often will be!).

To illustrate this point, Dennett seems likely to say 'yes' answer to Q1 (establishing that a theory attempts to directly be 'about' conscious experience) is a huge negative. I agree that a 'yes' to a Q1 and a 'no' to Q2 (meaning there is no causal-efficacy) is contradictory and therefore problematic. But I see a 'yes' to Q1 accompanied by a 'yes' to Q2 as a good thing: it's A1/A2-essential for consciousness 
Figure 4. Six Theories-of-consciousness on a Four-dimensional Map. (Coloured sub-domains are explained in Fig. 5). This Figure presents four-dimensional (Q1-to-Q4) map of Dennett's stance ('Den'), plus the 'Big Five' theories-of-consciousness ('IIT': integrated information theory; 'GNW': global neuronal workspace; 'HoT': higher-order thought; 'PC': predictive coding; 'OOR: orchestrated objective-reduction). Two-dimensional presentation of four data-dimensions: $y$-axis - manner in which theory is about conscious experience (Q1); $x$-axis - type of causal-efficacy attributed to consciousness (Q2); color - evolutionary advantage (Q3); shape - degree-of-detail in cortical/experience-relationship (Q4). (Table 2 explains Ra.b response-categories used on $x$ - and $y$-axes. See 'Key' for detail on shape/color-representations.) Q1 response-categorization: PC, HoT, IIT are explicitly promoted as theories of experience (placing them in the R1.4-category); GNW is interpreted in different ways in different places (leading to a R1.2/R1.3/R1.4 categorization); Dennett's stance excludes any reference to conscious experience per se (resulting in a $\mathrm{R} 1.1 / \mathrm{R} 1.2$ location). Q2 response-categorization: The five R2.1-located theories all implicitly set themselves in the sort of standard-physics setting (D2) that forbids any kind of causal efficacy. OOR is definitely causally-efficacious, but the kind of causality is unclear, because it may involve experience (leading to R2.3-allocation; Fig. 6C) or just a supra-brain computation (R2.4; Fig. 6B).(Q3/Q4 response-categorizations use data from Table 3, which is based on Section 10's analyses.) As discussed in Figure 5, the minimally-viable region for consciousexperiential science is the white area (at the intersection of R1.3 and R1.4 with R2.3). Only one theory (OOR) even reaches this area (and OOR currently lacks Q4's detailed cortical-relationships). Note also that only four of seven dimensions need to be mapped, because all theories discussed here neglect the three-dimensional theoretical terrain probed by Q5 to Q7.

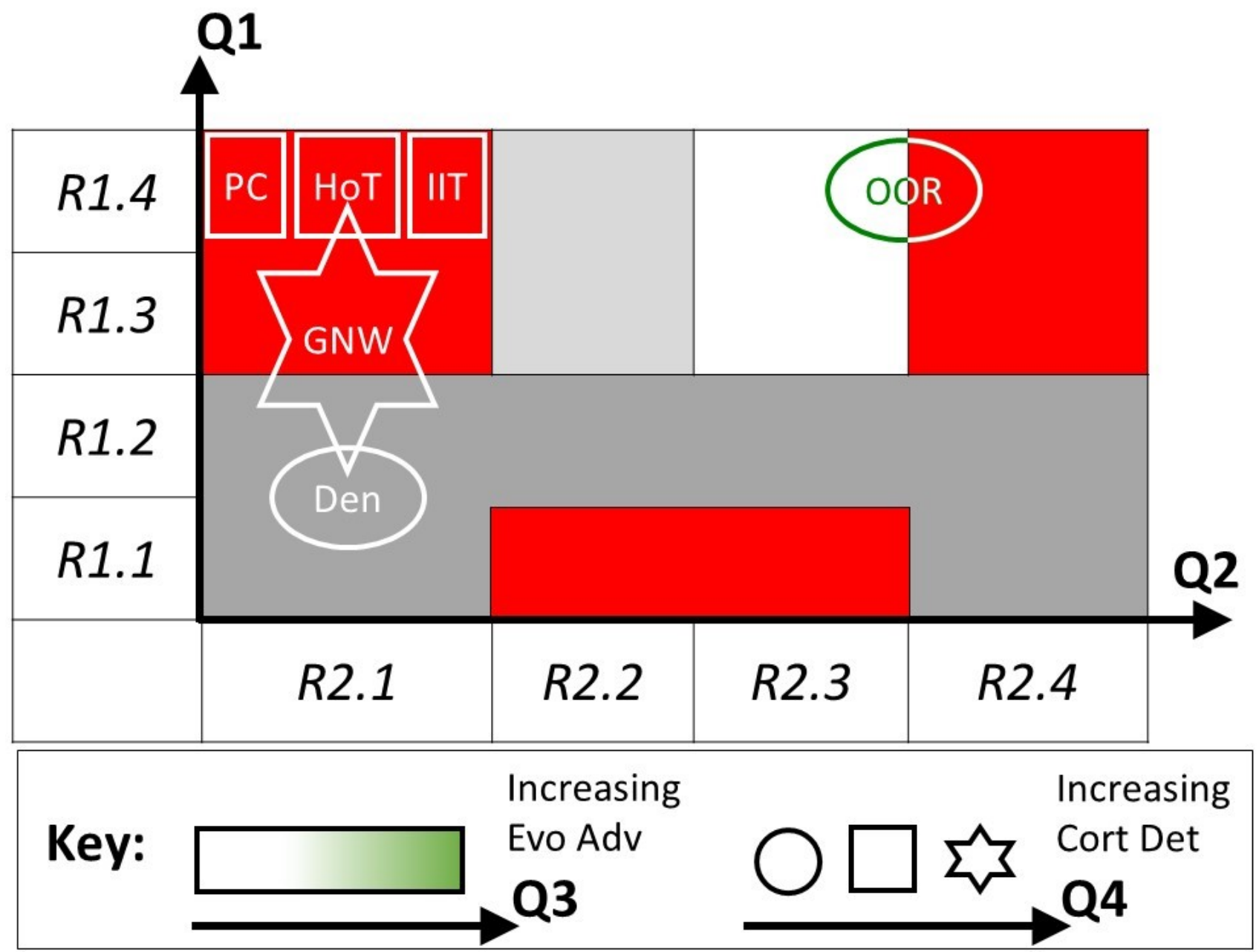


Figure 5. Two-Dimensional, Four-Answer-Category, Map Identifies Five Distinct Sub-Domains in Theory-Space. [Two-dimensional map: $x / y$-axes as in Figure 4.] Table 2's four-category answerstructure leads to identification of five qualitatively-distinct sub-domains that theories-ofconsciousness can inhabit [depicted as: red-' $\mathrm{X}$ ' (occurring in two places); red-' $\mathrm{X}^{\prime}$ '; light-grey-'I'; white-'I+II'; dark-grey-'N']. (1) Red-'X': scientifically-contradictory, concerning experience. These theories refer to 'conscious experience' (R1.3, R1.4) but make no allowance for causal-efficacy of experience (R2.1, R2.4), leading to contradiction under A2. (R2.4's causal-efficacy of consciousnessbeyond-experience doesn't definitively support scientifically-meaningful definition-of-experience). (2) Red-'X': scientifically-contradictory, concerning references to 'causal-experience'. These theories assert that experience is scientifically meaningless (R1.1), but R2.2/R2.3-definitions refer to experience. (3) Dark-grey-'N': not about experience. By construction, R1.1 and R1.2 explicitly label theories not-about-experience. (Under A1, theories having to exclude experience are less-preferred.) (4) Light-grey-'I': can support experiments-about-experience. Theories lying at the R1.4/R2.2intersection can be non-contradictory in defining/discussing experience, and can support scientificallyreliable experiments. (5) White-'I+II': experiments and evolution. The light-grey-'I' domain (type-4, immediately preceding) can't provide an evolutionary account of experience, because there is nothing that experience can add to the adaptive fitness of organisms. R2.3 allows for the possibility of experience-coupled supra-brain-computation (Fig. 6C), which could provide evolution-relevant experiential-contributions. The minimally-viable sub-domain for conscious-experiential science is the white 'I+II' rectangle (type-5, in the preceding list). Theories here are not contradictory (avoid red spaces), don't dismiss experience (avoid dark-grey area), and can support both a rigorous experimentalmethodology ('I'-labelled areas) and evolutionary-explanations for experience ('II'-label).

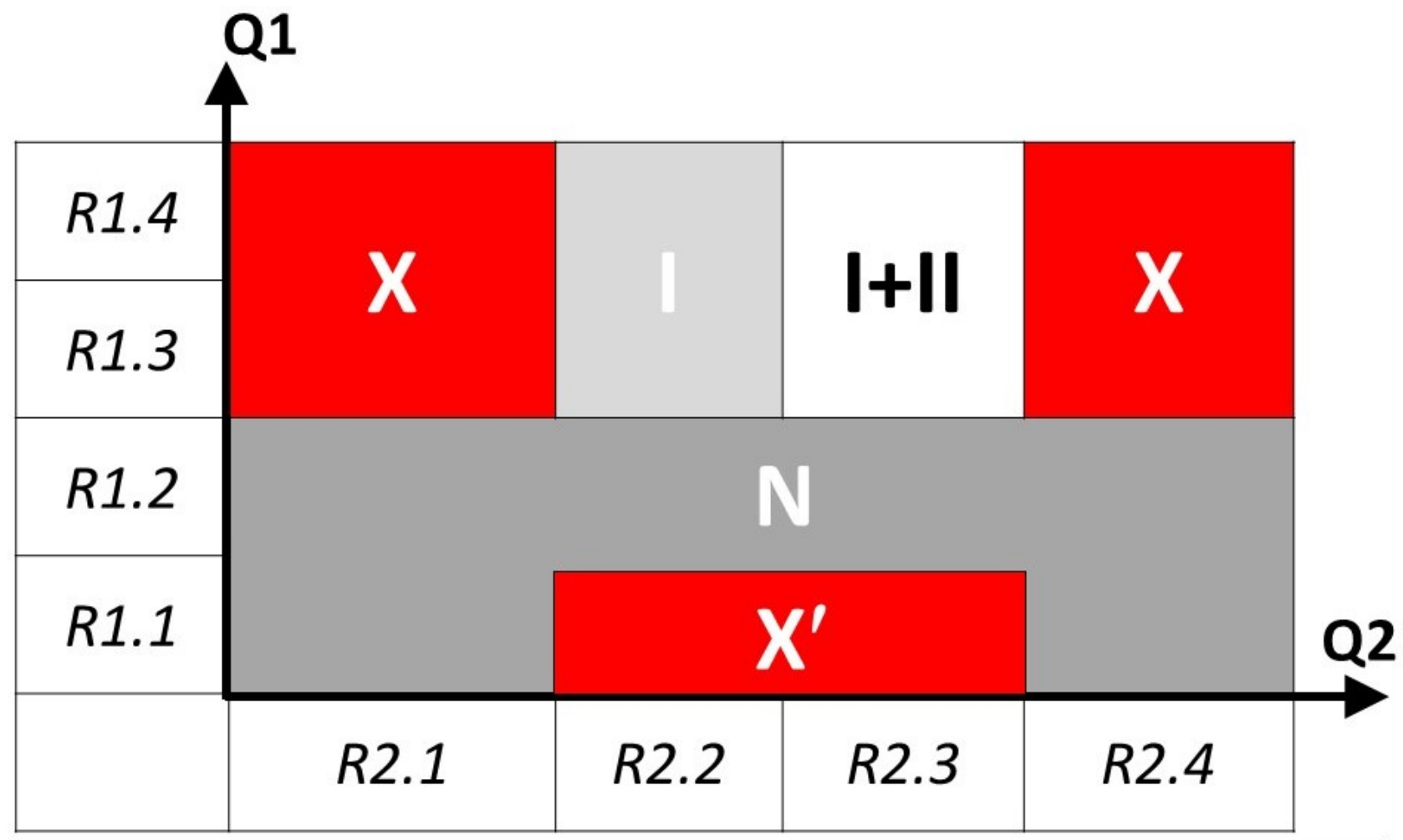

Key I: Experimentally-viable

II: Evolutionarily-viable $\mathrm{N}$ : Not about experience $\mathrm{X}, \mathrm{X}^{\prime}$ : Contradictory, Inconsistent 
science (if it exists!). Apparently, mainstream consciousness-scientists think that Q1-'yes'/Q2-'no' is problem -free [e.g. (Block et al., 2014; Storm et al., 2017)]. So it's possible to have three different evaluations of Q1-'yes'/Q2-'no': problematic, potentially-problematic (if we can't get to Q1-'yes'/Q2'yes'), and not-problematic!

If we end up back in the usual interminable debates, what is the point of structuring the dialogue using a question-inventory? Precisely because it draws out and pinpoints the fundamental underlying issues. One of the most frequent discussions I have about the structure of the field is with people who struggle to put Dennett's views into context. Q1 and Q2 do precisely that, exemplifying the value of structure in the midst of evaluation-disputes. (For more on Dennett's position, please see subsection 11.4.)

\section{Q1: Conscious Experience (Phenomenal Consciousness)}

\subsection{Q1: As Used in This Paper}

Q1 asks about the scope of the theory-under-consideration ('theory-X'):

Q1: Object-of-study. 'Does theory-X study and offer explanations for conscious experience?'

Of course, we need at least an orienting-definition of what's meant by 'conscious experience'. Following subsection 2.1.1, I use definition D1:

D1: Conscious experience. 'Conscious experience' is the collection of sights and sounds, body sensations and emotions, and 'inner' thoughts and imaginations, which exist in waking and dreaming, but disappear in deep sleep.

\subsection{Q1: Different Answer-Sets}

Subsection 2.3.1 mentioned the possibility of different structured answer-sets, thus essentially creating different versions of the inventory. Section 10's application of a simple inventory-version uses the set \{'yes','perhaps-sometimes','no'\} as the set of allowed answers for Q1. ('Perhaps-sometimes' means that there's either some ambiguity, and/or different accounts are given at different places/times.)

Figures 4 and 5 use the more nuanced Q1-answer set shown in Table 2. These answers allow for Denettian 'experience is scientifically-meaningless', as well as two kinds of access-consciousness, that do or do not claim to be about experience.

\section{3 'Consciousness' Not Limited to 'Experience'}

Chalmers' famous formulation of the 'hard problem' (Chalmers, 1996) separates experience from other aspects of mind. This is a mistake, because it asserts both that every function-of-mind is 'easy' to explain, in terms of conventional matter, and that function can be split from experience, in every case.

Q1/A1's prioritization of 'experience' over other aspects of mind [for example, meaning and understanding (Bayne, 2018)] is not meant as a hard-and-fast, everlasting, rigid, division, analogous to that made by Chalmers. To the contrary, as explicitly stated in Section 1, I see the 'consciousness' studied by consciousness science as an open-boundary topic, minimally including experience (if scientifically-possible). To add to Section 1's commentary, and amplify my critique of Chalmers' 'easy'/'hard' split, it may also be that experience is inextricably intertwined with certain functions - as discussed in the next Section! 


\section{Q2: Causal Efficacy (Dynamical Coupling)}

\subsection{Q2: As Used in This Paper}

Q1 asks about the causal-efficacy (or dynamical-coupling) of the theory-under-consideration:

Q2: Causal Efficacy. 'Does theory-X assume that contents-of-conscious-experience have causal efficacy, specifically in the sense that there can be transfer-of-information about the complete contents of experience, into brain-activity?'

This may seem like a curious question - but analysis elsewhere shows (Rosseinsky, 2018a) that causalefficacy is required for a conventional, reliable, experimental, science-of-consciousness to exist (A2). Relatedly, causal-efficacy is denied by the standard-physics setting that mainstream theories assume:

D2: Standard physics. 'Standard physics' means the current set of Standard Model fundamental-particles/fields and their couplings, together with general relativity as a theory of gravity, and/or any future developments of that theoretical base which specifically preserve its current exclusion of 'downward' experience-to-fundamental-particle couplings.

A2-requirement of causal-efficacy and its standard-physics denial obviously creates contradiction for current mainstream-theories (Rosseinsky, 2018a).

\subsection{Q2: Different Answer-Sets}

Section 10's application of a simple inventory-version uses the set \{'yes','partial','no'\} as the set of allowed answers for Q2. ['Partial' means that a theory proposes some kind of causal-efficacy, but it's not clear that complete information about the contents-of-experience can transfer into brain-activity. Lack-of-completeness is problematic for a science of experience, because it leaves open the possibility that report might not be reliably about experience (Rosseinsky, 2018a).]

Figures 4 and 5 use a more-nuanced answer-set, based on the possibility that novel (non-standard physics) dynamical/causal-behaviors associated with consciousness might be of two kinds. [Causality of either kind does not require dualism (Rosseinsky, 2018a).] The first kind is explicitly named in Q2: novel dynamics from information-transfer about contents-of-experience (Fig. 6A). Recalling Section 1 's 'experience-plus' definition of 'consciousness', the second kind derives from the possibility of computations-in-consciousness that are not executed by the brain (Fig. 6B).

For example, Orch-OR is associated with analyses suggesting that humans can execute non-algorithmic computations that their classical-matter brains cannot (Lucas, 1961; Penrose, 1989). Although these analyses are controversial [e.g. (Bringsjord and Xiao, 2000; Chalmers, 1995; Klein, 1995)], no definitive refutation of the possibility of supra-brain computation seems possible - beyond dogmatic faith in orthodox-physics!

Now write ' $c e$ ' for 'conscious-experience-related', and ' $b c e$ ' for putative components-ofconsciousness that are 'beyond' or additional to experience (and to the classical-matter brain), and ' $n s p$ ' for non-standard physics associated with causal-consciousness. Then, we can denote two basic causal-efficacy possibilities as 'ce-nsp' and 'bce-nsp'. Figures 4 and 5 use Table 2's Q2-answer-set, which essentially creates four options from by combining existence/non-existence of ce-nsp with existence/non-existence of bce-nsp (Fig. 6A-D). 
Figure 6. Different Kinds of Causal Coupling. ('ce-nsp' and 'bce-nsp' abbreviations are explained in subsection 4.2) A. Conscious-experiential coupling ('ce-nsp'). Brain-dynamics involved directly in conscious-experience (red spikes) associate with (black-dashed double-headed arrows) conscious experience (contents of black rectangle containing colored photo-image). Information-transfer to report-governing brain-dynamics (black spike) takes place (green arrows) both from dynamicsinvolved-in-experience, and from experience itself. (Horizontal dashed-line separates unseen and experiential realms; Fig. 1.) B. Beyond-conscious-experiential coupling ('bce-nsp'). In principle, 'consciousness' as defined in Section 1 can include computational actions that are beyond the capacity of the classical-matter-brain, schematically depicted here as the 'supra-brain computation' rectangle. One of many possible configurations is depicted: supra-brain activity takes experiential-configuration as input data (green arrow indicates information-transfer), and passes 'outputs' directly into braindynamics (contrast panel C). C. Maximal non-standard physics (ce-nsp plus bce-nsp). Here experience associates with brain-dynamics (as in panel A), and passes information to supra-brain computation (as in panel B). Now the 'outputs' of that computation are passed back into experience, and from there can influence brain-dynamics (via the experience/brain-coupling, as in panel A). D. Conventional view. In the conventional view of mainstream consciousness science, information-flow is one-way only - from brain-dynamics 'into' experience. Constructing a rigorous, reliable, science-of-experience is an apparently-insoluble problem for this view, as we can never know what's 'in experience' (Fig. 7)!
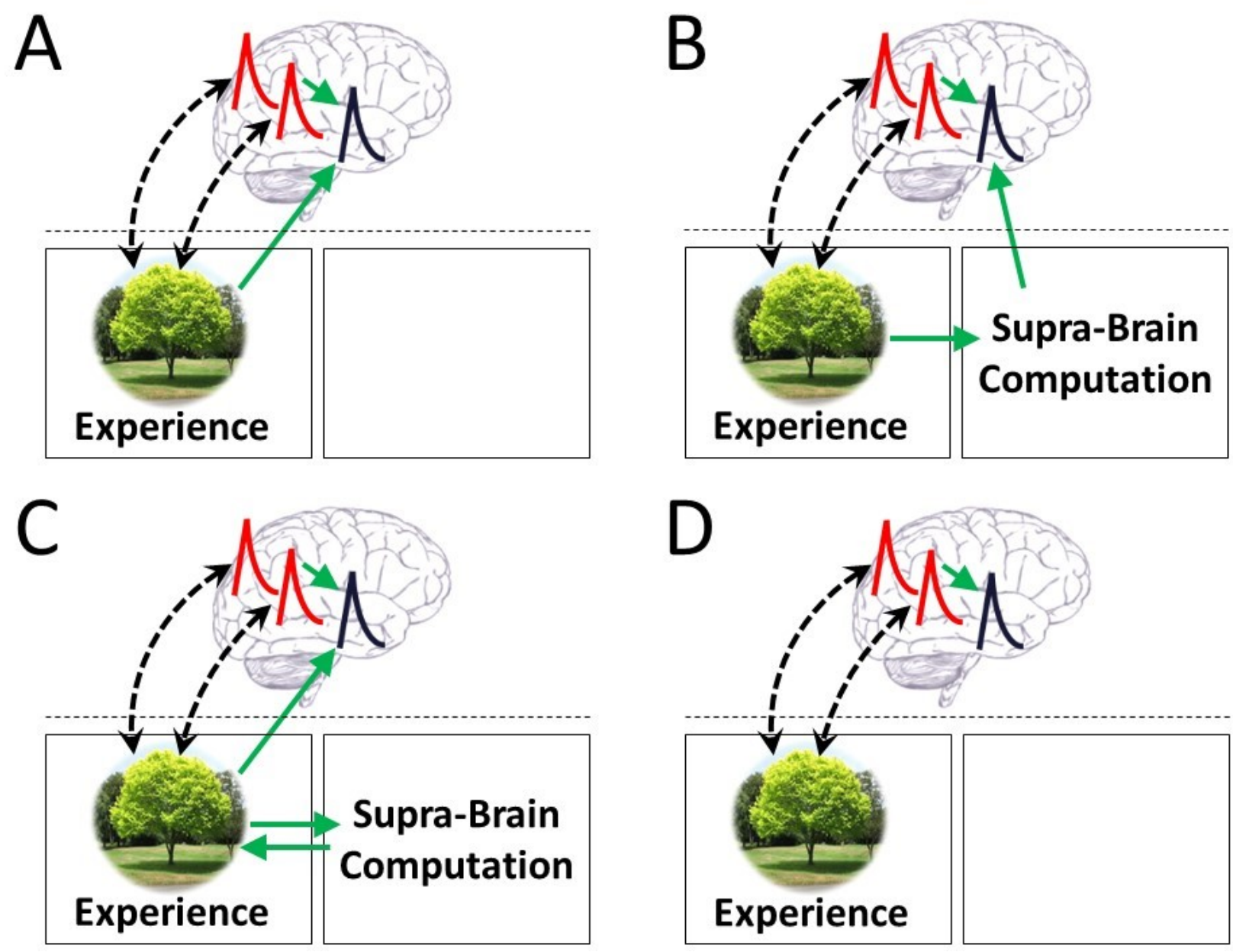
Table 2. A Four-response Structure for Q1 and Q2. For Q1 and Q2 (Table 1), the main thrust of the text uses a simple three-answer structure ('yes'/'partial-perhaps-sometimes'/'no'; e.g. see Sections 10 and 11). As subsection 2.3.1 points out, we can develop effectively-different versions of the questioninventory by changing the answer-structure, with questions held constant. (Relatedly, subsection 2.3.2 discusses the evolution of questions, over time.) Figures 4 and 5 illustrate the use of the alternative four-answer structure. (Figure 4 depicts use of the four-answer structure to map six theories-ofconsciousness. Figure 5 shows how the answer-structure decomposes the theoretical terrain in subdomains with different capacities and challenges.) The four-answer structure here is discussed further in subsections 3.2 (for Q1/R1.y) and 4.2 (for Q2/R2.y). (In terms of label-convention in the left-hand column, Rx.y labels the $y$-th response-kind for $\mathrm{Q} x$.)

\begin{tabular}{|c|c|}
\hline \# & Question/Response-Content \\
\hline Q1 & Object-of-study. 'Does theory- $X$ study and offer explanations for conscious experience?' \\
\hline R1.1 & 'No, and it claims conscious-experience is scientifically-invalid/meaningless' \\
\hline R1.2 & 'No, but it does study access-consciousness (without claiming report is about-experience)' \\
\hline R1.3 & $\begin{array}{l}\text { 'No, but it does study access-consciousness which is a report-of conscious-experience (and } \\
\text { perhaps other forms of access-consciousness)' }\end{array}$ \\
\hline R1.4 & 'Yes (and perhaps some versions of access-consciousness)' \\
\hline Q2 & $\begin{array}{l}\text { Causal Efficacy. 'Does theory- } X \text { assume that contents-of-conscious-experience have causal } \\
\text { efficacy, in the sense that there can be transfer-of-information about the complete contents of } \\
\text { experience, into brain-activity?' }\end{array}$ \\
\hline R2.1 & 'No, it lacks informationally-complete $c e-n s p$ and proposes there is no $b c e-n s p '$ \\
\hline $\mathbf{R 2 . 2}$ & 'Yes, it proposes informationally-complete $c e-n s p$, but no $b c e-n s p$ ', \\
\hline R2.3 & 'Yes, it proposes informationally-complete $c e-n s p$, and at least one form of $b c e-n s p '$ \\
\hline R2.4 & 'No, it proposes there isn't any $c e-n s p$, but it does propose at least one form of $b c e-n s p$ ' \\
\hline
\end{tabular}

\subsection{Logical Linkage between Q2-Causal-Efficacy and Q3-Evolutionary-Advantage}

As Section 5 will discuss, if we're to give a plausible account of Darwinian evolution of conscious experience, the natural world must contain both ce-nsp (the transfer of information from experience to brain-activity), and some form of bce-nsp that both contributes to organismic fitness, and is communicated to brain-activity via experience. This combination gives experience a direct-role in helping the organism thrive, survive, and mate. Under standard physics, experience provides no evolutionary advantage, because the entire evolutionary process can be accounted for by dynamics of atoms and molecules, never referring to 'conscious experience' (Rosseinsky, 2018b)!

\subsection{Possible Causal-Efficacy of 'Consciousness' Not Limited to 'Experience'}


Just as the conception of 'consciousness' isn't limited here to 'conscious experience', neither is the possible causal-efficacy of consciousness limited to influence-of-experience-on-brain-dynamics. (That much is obvious, from the explicit consideration of bce-nsp in the previous subsection!) But there are further nuances available. A beyond-brain computation could either couple directly with the brain, or it could pass computation-results into experience, and then experience/brain-coupling could transfer them into brain-dynamics. In this case, experience can play a pivotal role in function, invalidating Chalmers' alleged-division between the hard-problem-of-experience and easy-problems-of-function.

\section{Q3: Evolutionary Advantage}

\subsection{Standard Physics Explains Evolution without Reference to Conscious Experience}

Two views exist about the Darwinian evolution of consciousness. One emphasizes problems for conventional evolutionary explanations, for example claiming that conscious experience occurs too late to influence behavior, and therefore can't enhance fitness (Maley and Piccinini, 2018; Velmans, 2012). The other asserts that conscious experience has a biological function, for example in actionselection, and therefore can be selected for (Earl, 2014; Seth, 2018b).

A decisive and neglected argument points out that standard-physics (D2) can give an account of the entire evolution of all species, without making any reference to conscious experience (Rosseinsky, 2018b). Put differently, under standard physics, experience can't contribute to behavior - because that's already fully explained by physics (Elitzur, 1989; Huxley, 1893)! Thus, experience can't have actively contributed to evolutionary fitness at any point - and can't itself have evolved! (This argument isn't just a semantic trick. If experience has an evolutionarily-active role - as everyday first-person experience-of-experience seems to suggest! - it must add something dynamically, to standard physics.)

\subsection{Information-Processing Evolves, Experience Results: ‘Indirect’ Evolution}

One argument for evolution-of-experience under standard physics is that complex informationprocessing evolves (which is obviously reasonable), and experience then necessarily results from complex information-processing. I call this 'indirect' evolution, in contrast with a 'direct' path in which experience itself adds something directly to fitness.

The indirect argument for standard-physics evolution-of-experience seems plausible, at first sight. Certainly, if experience were a conventionally-emergent property (like temperature), it would work perfectly.

\subsection{Experience Isn't Conventionally-Emergent: Improbability of Indirect Evolution}

Unfortunately for the indirect evolution argument, experience isn't conventionally-emergent (Ellis, 2018; Rosseinsky, 2018b). As subsection 5.3.3 will discuss, this makes the indirect explanation astronomically improbable, to the point of scientific-implausibility.

\subsubsection{Experience Isn’t Conventionally-Emergent (I): 'Over and Above’ Character}

Conscious experience is 'over and above' the normal physical account of brain-dynamics, in the sense that electromagnetic field-activity, or the movements of molecules, aren't themselves inherently and obviously the same as 'experience of redness', or 'feeling of joy', or 'thoughts of "I think therefore I am"'. This doesn't mean that science can't explain experience (Goff, 2017), or that we need additional substances, or even that experience necessarily has a physically-fundamental character (Chalmers, 
1996). It just means that we need to establish an explanatory link between one aspect of reality (subsection 2.1.1's unseen realm!) and another (experience!).

The fact that experience has character (redness etc.) over-and-above unseen-realm-character (Fig. 1) means that it isn't conventionally-emergent. Conventional-emergence is often stated as the claim that 'experience is just like temperature'. Despite the fact that we experience temperature-related features in experience, temperature per se lies entirely in the unseen-realm (Rosseinsky, 2018b). Because conventionally-emergent explanations lie wholly in the unseen realm, they can't be the basis of unseen/experienced-realm relationships.

\subsubsection{Experience Isn't Conventionally-Emergent (II): Detailed Spatial Re-Mapping}

A second reason why experience is isn't conventionally-emergent lies in the detailed spatial remapping of information that's inherent in the brain/experience relationship. As explained in Sections 6 and 7, the spatial geometry of brain-encoded information about the external environment is very different from the geometry inherent in the re-presentation of that information as experience (Dennett and Kinsbourne, 1992). Thus, there needs to be detailed spatial re-mapping, to convert braininformation to experience. This kind of complicated transformation - without a physical substrate to implement it - lies outside the reach of conventional emergence. (Of course, temperature - and other conventionally-emergent properties - can vary by location. Bur that's not the same as having a complex, internal, spatial-structure. A complicated transformation function - that would also have to vary from individual, to account for anatomical variations - doesn't fit with a temperature-like explanatory-mode.)

\subsubsection{Experience Isn't Conventionally-Emergent (III): Long-List Emergence, Improbability}

Given that experience isn't conventionally-emergent, we are basically left with what I call 'long-list emergence' (Mukhopadhyay, 2018). (Other solutions are technically possible, including association of experiential states with fundamental particles. However, such solutions also lie outside presentlyorthodox physics.) In long-list emergence, the laws of physics contain statements such as 'human brains associate with experience', and 'when this aspect of brain-dynamics/configuration is in suchand-such a state, this aspect-of-experience occurs at that experiential-location'. The second clause ('when this aspect ...') turns into a (very!) long list, because it must explain brain-conditions for each possible contribution-to-experience. (E.g. the conditions for 'red-experience' here, and here, and here ... And then the conditions for 'green-experience', here, and here, and here ... 'middle-C-experience', here. and here, and here ...) These accounts can't appeal to general principles of neural coding, e.g. 'V1 encodes this sort of information, in that sort of map' - because e.g. neuroanatomical details can vary from person-to-person. Nor - as explained in subsections 6.5, 7.5, and 8.6- can mathematical formalisms [such as those inherent in IIT (Tononi et al., 2016) and other sophisticated approaches (Seth et al., 2011)] simplify the long-list (again, because they don't account for spatial re-mapping, and don't deal with neuroanatomical variations).

\subsection{Experience Must Couple, and Enable Supra-Brain Computation, For Evolution}

In the context of a standard-physics evolution-of-experience account, long-list emergence is either a complete mystery (in which case we don't have an evolutionary explanation for experience), or the list existed in the laws of Nature, e.g. at the Big Bang, and somehow the brain evolved itself - without any of the usual Darwinian guidance - into precisely the form that the long-list demands! Although this is possible, it's so astronomically unlikely (given the length and complexity of the list) that it's scientifically-implausible. 
However, because I prefer not to reason from conceptual-probability to truth (because there is no law of Nature I know of, guaranteeing non-occurrence of the astronomically-improbable), I declare an explicit assumption:

A5: Exclusion of Indirect Evolution. Indirect evolution of experience depends on long-list emergence, or evolutionarily-equivalent methods, which are excluded here on improbability grounds.

Standard-physics rules out direct evolution, and A5 excludes indirect evolution. This either makes the origin-of-experience a mystery, or, as discussed in subsection 4.3, demands that experience plays some explicit role in coupling the brain with a non-standard-physics dynamic which provides evolutionary advantage. Q3 asks if a theory contains that kind of evolutionary account:

Q3: Evolutionary Advantage. 'Does theory- $X$ provide a direct evolutionary advantage for experience?'

\subsection{Logical Linkage: Q2-(Total)-'No’ Implies Q3-'No'}

Logically (after A5), if Q2 establishes there is no causal-coupling of any kind from consciousness to brains, then Q3 must establish there is no direct evolutionary advantage for experience. Put differently, a Q2-'total-no' implies a Q3-'no'!

\section{Q4: Detailed Cortical-Relationships}

\subsection{Computational Neuroscience of Sensation/Perception vs Consciousness Science}

One distinction we have to keep clearly in mind is that between the computational neuroscience e.g. of sensation/perception (Bialek et al., 1991; Quiroga and Panzeri, 2013) and consciousness science. In principle, we might be able to understand and account for the complete computational/informational activity of the human brain, simply by understanding its wiring diagram (including its connections with sensory organs and the body), and the biophysical behaviors of its components (neurons, glia and so on). This investigation would of course be massively aided by observations and data from the 'live functioning' of the brain-system i.e. from human behavioral-experiments (that also collect braindynamical data), as well as e.g. from clinically-atypical cases, and animal studies.

So, what did the preceding paragraph describe? Computational neuroscience or consciousness science? If reports made in experiments are treated as being reliably about absence/presence of consciousexperience, then it's consciousness science. ['Report' isn't limited to verbal expression: it can include all sorts of behaviors (Timmermans and Cleeremans, 2015).] If there's no experience-related attribution/interpretation, it's computational neuroscience.

\subsection{Reports: About Experience ... Or Not?!}

To clarify any lingering misunderstandings concerning the distinction between reports-taken-to-beabout-experience, and reports-that-are-just-reports, consider a report about some external environmental feature, a colored edge, say.

As a general point, it seems clear that there is information encoded in the brain that's not represented in conscious experience. [Note that here, as in many other places in this paper, this statement claiming knowledge about what is and isn't represented-in-consciousness presumes currently-untested existence-of-causal-coupling (A2). In order to discuss issues for a putative, future, science-of- 
experience, my approach is to talk as if future experiments will demonstrate the existence of such coupling. This is methodologically OK, provided I declare the future-experiment-contingency - and with the understanding that if future experiments disprove causal-coupling, all discussions here about a science of conscious experience are invalid!]

Thus, report could in principle be related to either information-represented-in-experience, or information not-available-for-experiencing. We can't infer merely from report about the colored edge, that it was (or wasn't!) represented-in-experience. [Of course, we can ask people: was that report based on experience? The scientific issue is that, if they say 'yes' or 'no', for such answers to be scientificallydependable information-about-what-experience-contains must be passed into brain-dynamics. That's forbidden by standard physics, however, leading back to Q1/Q2 tensions for mainstream theories-ofexperience.(Rosseinsky, 2018a).]

Again, if we $d o$ distinguish between reports we consider to be about contents-of-experience, and reports of information-that-isn't-represented-in-experience, we're doing consciousness science. Otherwise, we're doing computational neuroscience. Clearly, the two are intimately related (if the former exists as a science!). But they're not the same.

\subsection{A Complete Neuroscience-of-Encoding is Not a Theory-of-Experience}

It can be easy to confuse progress in understanding how the brain encodes environment, body-state, decision variables, strategies, choice, and motor/somatic programs, with progress in understanding conscious experience. According to the so-called 'naturalization-of-consciousness' program, everything that we consciously experience has a basis in brain-activity. So, a pre-requisite for conscious-experiential science is understanding brain-encoding. (I use 'encoding' throughout to include not just representation-of-environment/body, but also action-related constructs). However, understanding brain-encoding isn't the same as creating a theory-of-experience.

If there was only one way in which the brain encoded environment, body-state, and so on, the simplistic theory-of-consciousness suggested in subsection 2.4.2.1 could actually be a single, consistent, hypothesis! 'Everything encoded by the brain appears in experience' (Fig. 7A). But even then, there would be many possible maps from encoding to experience - and many possible experiential states that could be associated with a given brain-state (Fig. 7B-C), leading to different, competing, subhypotheses of the simplistic-theory. Different experiential-states/theory-versions can all support the same data-reports: it's just that the same report would or would not actually be about experience, in different ways under different theories (Fig. 7B-C). [In empirical-actuality (Fig. 7D), there are many ways in which a single percept is encoded in the hierarchical/distributed-brain (Rosseinsky, 2015), making the problem even more complicated: 'everything encoded by the brain appears in experience' could actually result in many different representations of reality appearing at the same time!]

\subsection{Theories-of-Experience Must Establish Detailed Encoding-to-Experience Relationships}

So, having a complete neuroscience (a complete understanding of the encoding-brain) is not the same as having a theory-of-experience: there's more work to do. Of course, that seems to be recognized by the very fact that we have different theories-of-consciousness, incremental in different ways to computational neuroscience. But there's a difference between a general recognition and a detailed one.

What do I mean by a 'detailed' recognition? Say we had a complete understanding of how the brain encodes color in the visual scene. A detailed theory of consciousness doesn't just say 'the brain encodes color, so therefore we can be conscious of color' (a general statement!). It says: 'when this particular 
Figure 7. Simple Theories of Conscious Experience. A. A simple 'only-one-encoding' theory-ofexperience. [Dashed-horizontal line separates upper unseen-realm from lower experiential-realm (Fig. 1).] Vertical/horizontal line stimuli (purple lines) participate in generating experience-involved brain dynamics (red spikes), which are associated-with (black-dashed double-headed arrows) corresponding components-of-experience (black lines). Information is transferred (green arrow) from experienceassociated brain-dynamics to report-governing dynamics (black spike). A complicated series-of-events (not shown; depicted schematically by green-dashed arrow) leads to experience of a verbal, spokenout-loud, report 'I see a vertical line'. B, C. Two alternative theories-of-experience. Even if each unseen-reality feature is only encoded in one simple, known, way, multiple theories-of-consciousness can be stated. In panel B, experience only occurs for vertical edges, and not horizontal ones; in panel $\mathrm{C}$ the converse holds. In both cases report-of-experience is exactly the same, because it is governed by brain-dynamics, not experience! The objection 'we know neither B or C happens' itself presumes information-transfer from the internal-structure-of-experienced-reality (below the dashed-line) into brain-dynamics (above the line), as in Figure 6A. This requires non-standard physics. (Whether such non-standard dynamics exist is properly an empirical question.) The takeaways are, even under the simplest possible encoding-schemes: (1) theories-of-experience are more than theories-ofcomputational-neuroscience; and, (2) there's no conventionally-reliable consciousness-experientialscience without experience-to-brain information-transfer. D. Illustrative multiple-encoding problem. Even a single, simple, unseen-realm stimulus (purple vertical-line) is of course represented in braindynamics in many (possibly subtly-different) ways. Then, 'experience replicates encoding' leads to many theories-of-experience (or, one theory in which percepts appear many different ways)!
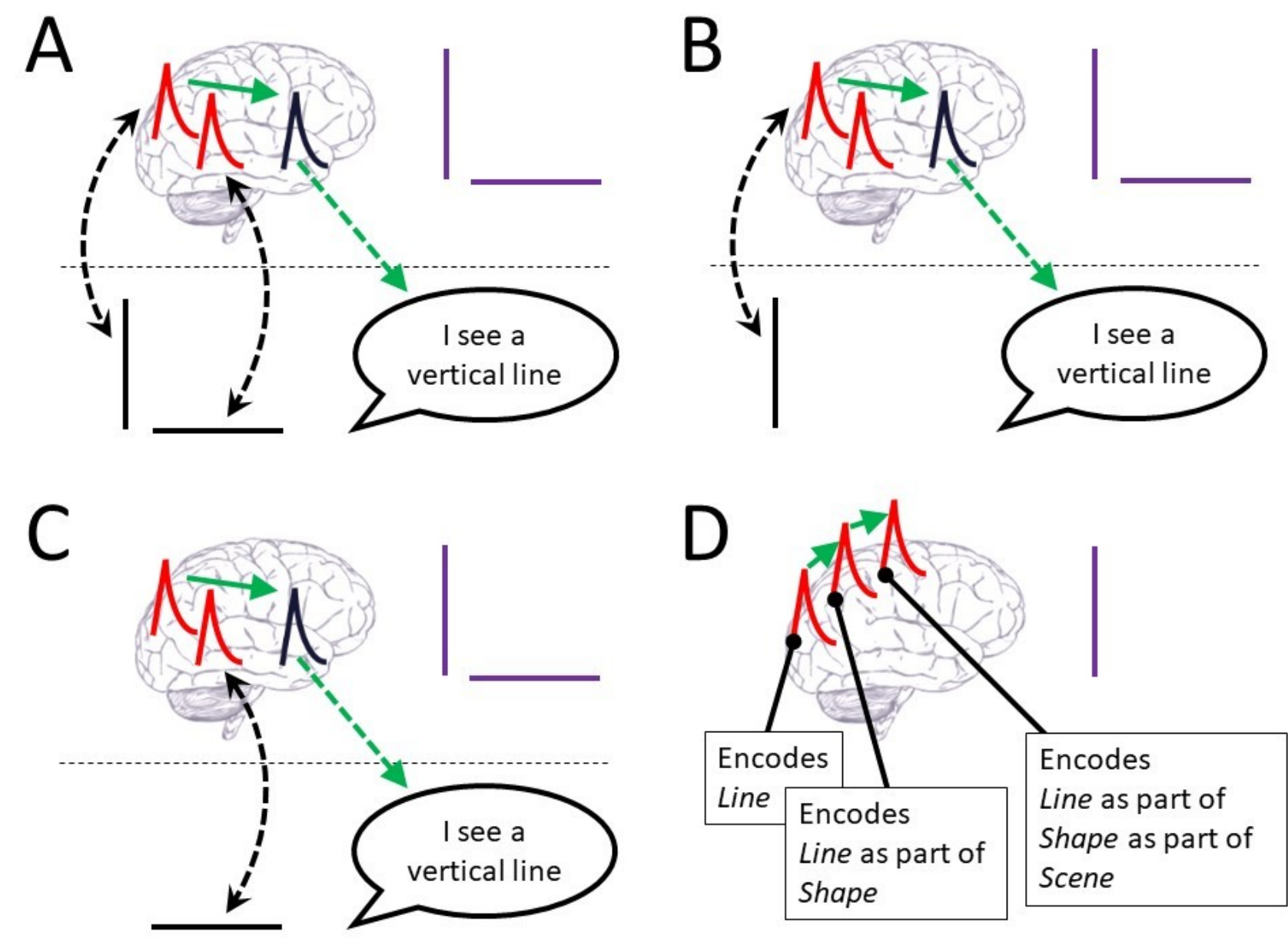
neuron (or set of neurons) are in this particular dynamical state, this particular shade of red occurs at this specific experiential-location'. A theory-of-conscious-experience must tell us precisely and in detail how to associate experience with brain-states. Even if computational neuroscience tells us what encoding states are about, we still need to write a detailed set of rules for the encoding-to-experience translation:

Q4: Detailed Cortical-Relationships. 'Does theory-X state or create detailed, explicit. relationships between components-of-experience, and cortical-activity that encodes specific aspects of body/environment, and/or governs specific behavioral/somatic activities?'

\subsection{The Rewiring Thought-Experiment}

The point behind Q4 is: how does Nature 'know' which component-of-experience to associate with a specific encoding-dynamic? When we develop theories-of-reality, we're putting ourselves in Nature's shoes, so to speak. When we reason that 'computational neuroscience knows what this encoding dynamic means ... which tells Nature what experiential-component must be associated with it', that implies that the entire understanding of neuroscience (including specific neuroanatomical wiring of each human!) is used by natural law. This would be a radical revision to the current view in physics, which says that Nature knows only about present-moment fundamental field/particle states, and recomputes the next instant of unseen reality (Fig. 1) from those states, and mathematical law. All of unseen reality, at all levels - up to, including and beyond brains - is accounted for by that natural process, in the current structure of scientific law.

To illustrate this point, considering the following thought-experiment. Rewire the brain of a human experiencer so that some of the neural inputs from the ears are re-routed into the visual system, and some of the neural inputs from the eyes are re-rerouted into the auditory system.

What happens to experience? Does it stay unchanged - because Nature 'knows all the wiring details and uses that knowledge to figure out which experiential-components to post into experience'? Or does part of visual experience now respond to sounds, and part of auditory experience respond to visual stimuli? Conceptually, both are possible. But a theory-of-consciousness has to say which. Experimental data [e.g. direct cortical stimulation (Desmurget et al., 2009; Parvizi et al., 2012)] tends to suggest it's the latter, switching-of-experience, option that would occur. But that means that individual neurons (or specific groups of neurons) are specifically-hardwired to certain components-of-experience. That would mean that a theory-of-consciousness must list all those linkages, in detail!

\section{Q5: Locality/Geometries}

\subsection{A Detailed List Highlights the Locality/Geometries Problem}

If a theory-of-consciousness does step up to Q4's detailed list, another problem typically results, immediately. To demonstrate, I'll use very simple theories-of-encoding and consciousness. But mathematically-complex [e.g. (Seth et al., 2011; Tononi et al., 2016)] or physically-sophisticated [e.g. (Hameroff and Penrose, 2014)] theories do not escape challenges implicit in Q4-to-Q7, as straightforward examination would reveal.

Consider a simplified, demonstrative, pedagogical, theory-of-encoding in which the firing of neurons one, two, and three encode the existence of a red-stimulus at respective-locations one, two, and three in the external environment of a human-experiencer (Fig. 8A). (This simplified model uses all-ornothing neuronal-firing, and neglects multi-unit encoding of color, for the moment - but see subsection 
Figure 8. Locality and the Three Geometries. A. A simple theory-of-encoding. In a simple place/rateencoding, specific neurons at specific brain-loci encode the presence/absence of red-stimuli (purpleedged red-circles) at specific environmental locations, by firing (red spikes) or not-firing, respectively. (Note that mathematically-complex theories such as IIT do not escape points made here: in fact, they are more prone to locality critiques than simple pedagogical models.) B. Brain-geometry doesn't recapitulate environmental geometry. Diagonal dashed-line now separates unseen-realm (upper-right) from experiential-realm (lower-left). If experience (borderless red-circles, lower-left) follows braingeometry, it doesn't recapitulate environmental-geometry (arrangement in unseen reality, upper-right). (For 'recapitulation', see panel D.) C. Complex folding of primate cortex. This image reminds us that in reality, the geometrical issue is not 'straight-lines $v s$ triangles' as in panel B. Otherwise-orderly topographic maps are folded into complex 3-D arrangements, in biological brains. D. Recapitulation. Recapitulation-of-geometries is the same concept as similarity-of-shapes. Here $\left\{\mathbf{r}_{i}^{\prime}\right\}$ recapitulate the $\left\{\mathbf{r}_{i}\right\}$ (and conversely), even though scales/orientations differ. E. The locality problem. To achieve recapitulation in panel $\mathrm{B}$, there must be at least one non-local transfer of information (as indicated by the black arrow, moving the topmost 'experience-of-redness' from its original, red-dotted, location). F. Experiential geometry differs from both brain-encoding and environmental geometries. Familiarly, parallel lines in objective reality (straight lines at constant measured-separation from each other) converge in visual experience (red-dashed lines). This is not just an artistic or photographic artefact. Thus, visual experience relates three geometries, with two immediate problems: locality (panel E), and the transformation from brain (or environment!) to experience - where in the natural world are the details of this very specific, Euclidean-to-hyperbolic, transformation implemented, or encoded (Q4)?

A
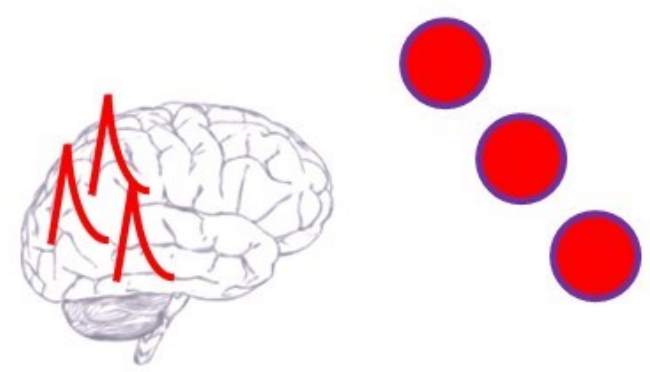

Solomon SG, Rosa MGP. A simpler primate brain: the visual system of the marmoset monkey. Front Neural Circuits (2014) 8: doi:10.3389/fncir.2014.00096
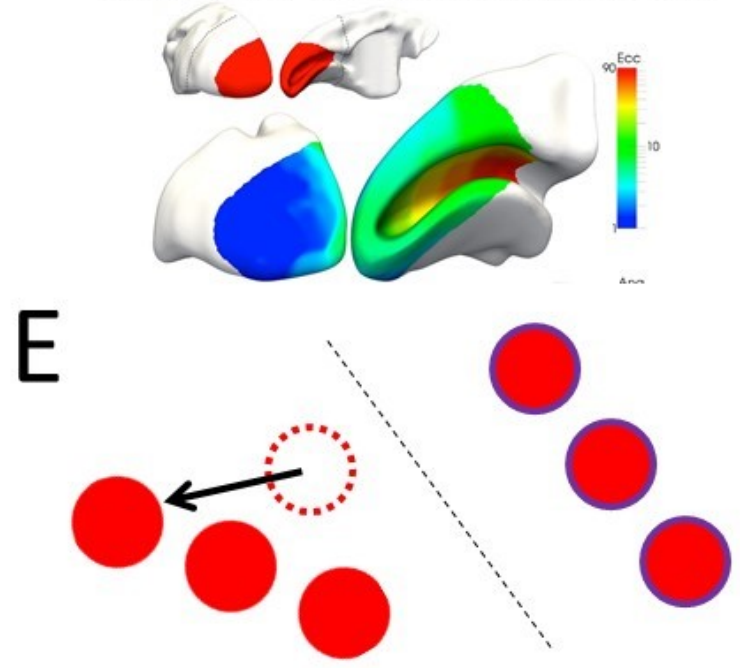
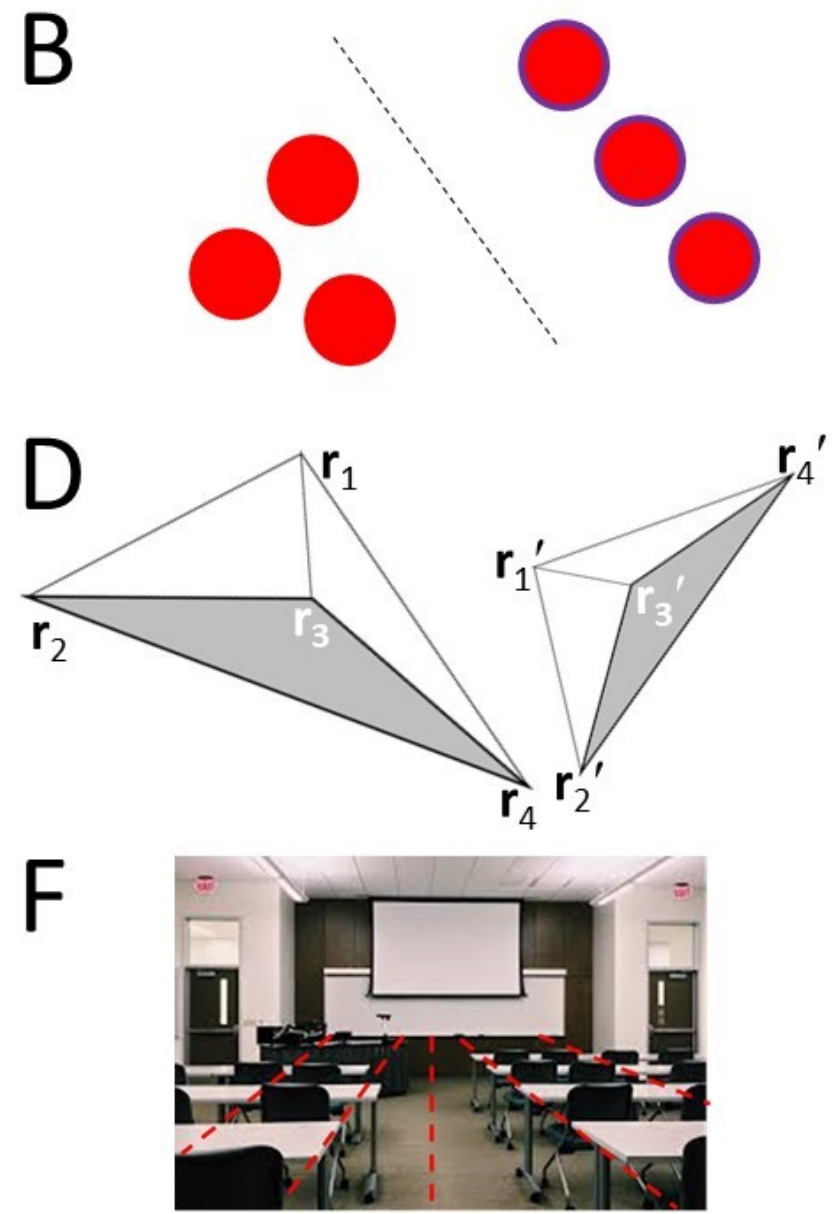
7.2.) Immediately we have two different geometries (Fig. 8B-C): the relative arrangement of environmental-loci, and the relative arrangement of brain-encoding-loci. These relative arrangements need not be the same, and in fact neuroanatomically we know that they aren't, typically: there is no brain-area where encoding-neurons are arranged in a 3-D manner that recapitulates environmental geometry (Fig. 8D).

Next, look at a simple theory-of-consciousness that says 'experience of red at a given environmental location is identical to the firing of that location's red-encoding neuron'. This would lead to an experience-of-redness that wasn't arranged in the same way (or 'didn't recapitulate') the external environment. For example, in early visual processing (Fig. 8C), the 3-D environment is typically encoded in two sheet-like areas folded in a complicated way, on the cortical-surface. (This means there are actually at least six encoding-neurons for three environmental-loci!) So, a local theory (experience local to encoding) leads to problems for identity-like theories, because encoding isn't arranged as the environment is (Fig. 8E)!

Finally, things are actually worse than this! There's a third geometry involved, namely the way that subjective experience is arranged - which is not the same as objective, environmental, geometry (Wagner, 2006). (As usual, this reference to facts-about-experience is contingent on Q2's causalefficacy.) For example, parallel straight lines in objective geometry converge in experiential geometry. Thus, the real goal is that encoding-neurons recapitulate experiential-geometry, not environmentalgeometry (Fig. 8F). This is 'worse' because somewhere in Nature we should find this additional transformation (brain-encoding geometry to experiential-geometry). Not only does brain-encoding geometry fail to recapitulate experiential-geometry (in the same way that it fails to recapitulate environmental geometry), but also, we now need Q4's detailed-list to account for the brainencoding/experiential-geometry transformation (or, we need to find that transformation implemented, encoded or embodied somewhere in physical reality).

Of course, we can get around non-recapitulation by just saying 'experience is arranged spatially in a different-way than encoding is'. But that means non-local physical action: neural encodinginformation associated with conscious-experience is spatially-relocated to create the right geometry. This minimally seems to require relaxation of conventionally-local transfer-of-information in orthodox physics. Of course, relaxations are permissible, given pressing conceptual or (preferably!) data-based reasoning. The primary issue is that one of the foundational dogmas of mainstream consciousness science seems to be 'we can explain consciousness without varying orthodox physics in any way'. [For example, the terms of reference of the recently-launched journal Neuroscience of Consciousness (https://academic.oup.com/nc/pages/journal_policies) exclude 'alternative physical foundations' although a definition of 'non-alternative' foundations isn't given anywhere! The problem of hazy dogma is by no means limited to this case, which is simply included as a particularly clear-cut example.]

These issues lead us to Q5:

Q5: Locality/Geometries. 'Does theory- $X$ offer an explicit solution to the tension between orthodox-physics locality, and the difference between brain-encoding geometry and experiential geometry?'

\subsection{Multi-Unit Non-Locality}


As is well known, one inaccuracy in the preceding subsection's model is that early-visual-processing encodes color at a single environmental location in a vector code of the firing-rates of three neurons, not in the firing rates of single-neurons.

But this is just another spatial-locality problem for every theory-of-consciousness that draws on any analogous multi-unit codes. If a theory-of-conscious-experience has to assimilate information from multiple brain-locations to compute the appropriate contents-of-experience at a single experientiallocation, this must involve spatially non-local information-transfer, in the absence of additional explanations. [This kind of non-locality is practically the basis of mathematically-sophisticated theories (Seth et al., 2011; Tononi et al., 2016).]

\subsection{Temporal Non-Locality}

Another locality-problem concerns time. For example, the assessment of a firing-rate requires finite time-interval. This means that the natural action involved with instantiation or association of experience can't act instantaneously (as all other natural actions do).

Objecting that 'cortical encoding doesn't use crude firing-rate codes' doesn't avoid temporal nonlocality: relative spike-timing codes (Quiroga and Panzeri, 2013; Rieke et al., 1997) also need finitetime data-collection for their decoding. Nor is there any refuge in the claim 'experience just isn't like that - it doesn't depend on a "natural action". It's clearly not conventionally-emergent (subsection 5.3). This doesn't mean experience is beyond 'naturalization' - but we must take the nature-of-thephenomenon seriously (Section 1's second 'ground'), if we want to do rigorous science (the first ground!). Experience is a natural phenomenon with a rich internal structure that depends on distilling information from brain-activity that's distributed across space and time. How does this happen, in the absence of brain-additional physical-structure to perform that distillation? If we say 'it just does [happen]', the details of that 'happening' become content in a theory-of-consciousness. If that content violates spatiotemporal locality - let's just acknowledge that.

\subsection{The Binding-Problem-for-Consciousness Accentuates the Locality/Geometries Problem}

Finally, the binding-problem-for-conscious-experience (Cleeremans, 2003; Feldman, 2013; Singer, 2001; Treisman, 2003) is just yet another locality/geometry problem. Here the issue is that e.g. sound is encoded in one brain-area, and vision in another (Fig. 9A). Importantly, for all non-locality issues, there's no 'Cartesian Theater' in the brain - a single brain-area where all sensory-modalities converge into a set of experiential-geometry-recapitulating neurons, whose individual-member's activities singly encode all perceptual-features at individual experiential-locations. Mechanisms like synchrony or coherence [(LaRock, 2006; Melloni et al., 2007; Varela et al., 2001); Fig. 9B] don't solve the need to aggregate information at spatially-separate brain-locations, to compute or instantiate a componentof-experience at a single experiential-location (Fig. 9C). Synchrony/coherence-induced phase might indicate which spatially-separate encodings 'go together' - but in the absence of a Cartesian Theater, there still needs to an additional natural-action to receive and interpret phase-information, and use it in the instantiation of experience (Fig. 9D).

\subsection{Why Mathematically-Complex Theories Don't Escape Locality Problems}

One way to try to sidestep content of previous subsections is to claim that 'the mathematical sophistication of theory- $Y$ makes it so different from the simple firing-rate/identity example, that locality/geometry problems don't apply'. This is simply incorrect. 
Figure 9. The Binding Problem for Conscious Experience. A. The Feature-Binding Problem. As is well known, different feature-types (e.g. sound and vision) are primarily encoded in spatially-separate brain-areas. In the absence of a single brain-area in which all encoding-activity converges (e.g. a 'Cartesian Theater'), this leads to two different binding-problems, for informationprocessing/behavior, and for conscious-experience. B. Synchrony/coherence can solve informationprocessing/behavioral binding-problems. Inset: Neurons at $\mathbf{r}_{1}$ (auditory processing) and $\mathbf{r}_{2}$ (visual processing) are pre-synaptic to a neuron with axon hillock at $\mathbf{r}_{3}$. (Red lines schematically depict 1-to3 and 2-to-3 axonal connections.) Firing at $\mathbf{r}_{1}$ and $\mathbf{r}_{2}$ (red spikes), synchronized with LFPs (black waveforms), can lead to supra-threshold summation of excitatory post-synaptic potentials (red semi-circles) at $\mathbf{r}_{3}$. (This is a reminder of well-known roles that synchrony/coherence can play in neural computation at physically-connected loci.) C. Binding-problems for conscious experience: experience embedded in matter-space. (Diagonal dashed-line separates upper-right unseen-realm from lower-left experiential-realm [Fig. 1].) Theories-of-experience must say where experience is, relative to braindynamics. In one solution-style, experience is embedded in the same space as the matter-brain. Synchrony can't solve locality/non-recapitulation problems (Fig. 8E): auditory experience (schematically depicted by note-symbols) and visual representation of the actual-origin-of-sound (schematically depicted by black-square) are separate in experience, if local to brain-encoding dynamics. D. Binding-problems for conscious experience: experience embedded in 'virtual'-space. To avoid panel-D problems, some theories use a 'projection function' $\Pi$ (purple arrow), creating experiential-contents in 'virtual'-experiential space. This approach can use e.g. phase of LFPs to compute co-location (but leads to other problems e.g. for multiple experiencers: Section 8 and Fig. 10).
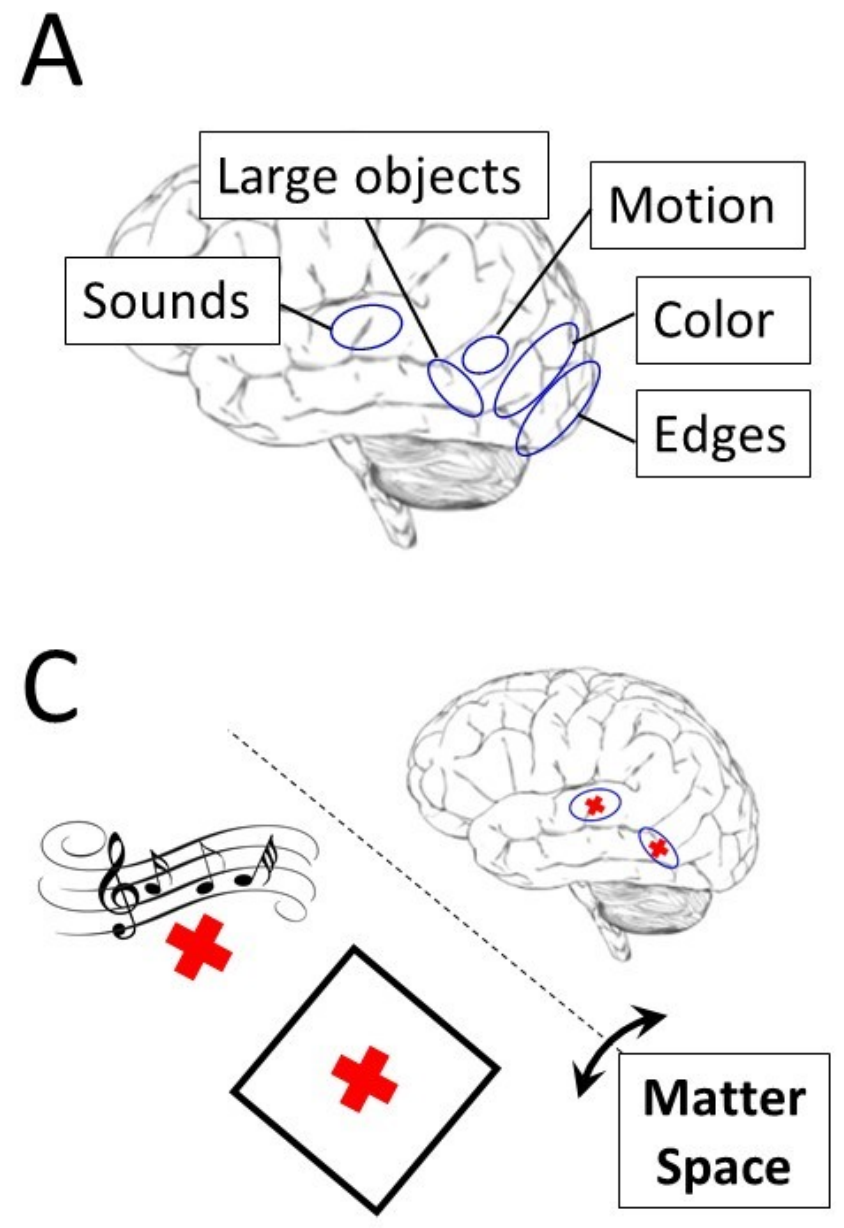
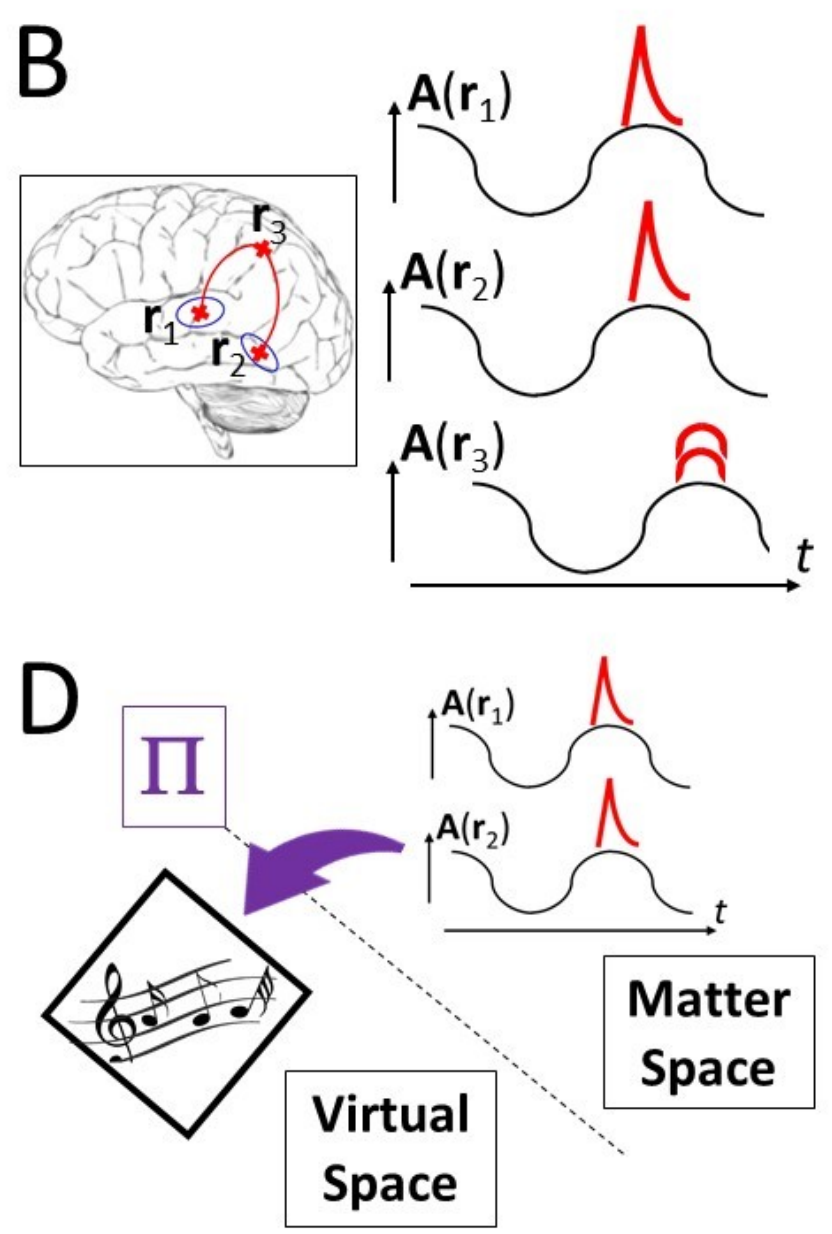
Consider a theory that proposes the contents-of-experience depend on some complicated, multilocation formula [e.g. (Seth et al., 2011; Tononi et al., 2016)]. Right away, such formulae presume nonlocal aggregation of information! Then, they must draw on multi-location encoded-data (color, edges, shapes, sounds, etc.) if details of experience are to be grounded in brain-encoded data. They may use more sophisticated e.g. spike-timing encoding-theories. But encoding-data is still distributed over space and time.

\subsection{The Curious Case of Locality/Geometry: 25-Years Ignored?}

Analyses here are really a cartoon sketch of detailed and comprehensive treatments of space in theoriesof-consciousness, which have only recently been advanced (Rosseinsky, 2019, 2018b). Nevertheless, the pivotal absence of the Cartesian Theater (subsection 7.4) - and its orthodox-physics implications - have been known for more than twenty-five years (Dennett, 1991). Directly, if we rigidly assume locality and entailment features of orthodox physics, the relationship between brain-activity and experience cannot be explained, because of the divergence between experiential and brain-encoding geometries. (The 'entailment' feature of orthodox physics is that any details of any systems-level natural-action must be traceable to a physical structure. Thus, the detailed rearrangement of brainencoding into experiential geometry should have some correlate in physical reality - a structure that implements or executes that re-arrangement. But the project of mainstream consciousness science is to explain the brain/experience-relationship precisely without any structure over-and-above that of the brain - which doesn't currently appear to contain any information about experiential-geometry!)

Why has consciousness science ignored locality/geometry problems for so long? Plausibly, the mainstream field holds to two dogmas: we will, without doubt, explain conscious experience (Block et al., 2014); and, that explanation will take place in an orthodox-physics framework (https://academic.oup.com/nc/pages/journal_policies). Locality/geometry-problems deny the joint viability of these dogmas. (If currently-orthodox physics holds, locality/geometry says we can't explain experience. If we are to be able to explain experience, locality/geometry says we can't sustain currently-orthodox physics.) In fields with sociological problems (Smolin, 2007), dogma-denying 'heresies' are simply suppressed, repressed, denied, and ignored. But such fields aren't really doing science, anymore.

\section{Q6: Lawfulness/Individuated-Multiplicity}

\subsection{Spatiotemporal Homogeneity of Physical Law}

I use the term 'homogeneity of physical law' to label the presumption that the same cause, at different times or in different places, has the same effect. (People who work in dynamical systems don't like 'cause/effect' language - they prefer to talk about 'coupling'. In this language, the same system - in different locations or at different times - has the same couplings. Of course, in both cause/effect and coupling languages, we assume 'all-else-equal', concerning the context into which a cause/system is relocated.) It's (presumed!) homogeneity of law that allows us to reason about the properties and behaviors of electrons in distant stars, based on understanding of those properties and behaviors in a terrestrial laboratory.

\subsection{Lawful Dependence of Experience on Brain-state}

Say we solved all problems raised by Sections 2 to 7, and had a theory-of-experience that associated specific brain-states with specific experiences. Following subsection 6.3, this must be more than a complete computational-neuroscience-of-encoding. Following Section 7, it must contain a detailed 
explanation of relationships between activity at specific brain-locations and components-of-experience at specific experiential-locations. For example, 'activity $A$ at brain-locations $B$ leads to experientialcomponent $E$ at an experiential-location $F$ ' (Fig. 10A).

\subsection{Homogeneity and Multiple Experiencers}

In Section 7, we saw that the way the brain encodes experience means that we can't have a spatiallylocal theory-of-experience, in which an experiential-location $F$ is essentially the same as a brainencoding location $B$ (Fig. 9C). Therefore, we need to come up with alternative explanations for spatialrelations between brain and experience. Instead of using the 3-D space that contains brains themselves (in some ontological conceptions), one idea is to think of experiential-space as an ontologically-junior construct (Fig. 9D) that's instantiated by specific brain-dynamics. (For example, this space is created on waking, and disappears in deep sleep. The D1-definition of experience focused on contents of this space.)

A basic structural feature in many theories of this kind is to think of certain stereotypical brain-locations as being critically-significant to experience: stereotypical activity in these locations leads to 'posting' specific experiential-components to specific locations in dynamically-created experiential space. (This approach already has a problem in cohering with orthodox physics: Nature has to assign special status to certain locations based on their neuro-anatomical or neuro-functional significance. With regard to the seven-question inventory, this issue is really related to Q5, specifically whether the way in which detailed cortical-relationships are created respects the structure of current natural law.)

What I've just described is of course just a specific, posting-into-virtual-experiential-space, implementation of ' $A$ at $B$ leads to $E$ at $F$ '. But this specific implementation has consequences! If physical homogeneity-of-action holds, then each $A$ at any stereotypical- $B$ should lead to the same $E$ (which is fine) at precisely the same $F$ (which is not fine!) (Fig. 10B). It means that $A$-dynamics in different brains - of multiple experiencers - will lead to $E$-components at one-and-the-same $F$-location (Fig. 10C). (This sort of thing typically doesn't happen when orthodox physical-space is used to embed experience, because experiences associated with different brains are spatially-close to them, keeping experiential-spaces separate and distinct; e.g. see subsection 8.7.2.)

\subsection{All Experience (Of All Experiencers) Occurs in One, Common, Experiential Space?!}

The logical corollary of the posting-into-virtual-experiential-space scheme (if orthodox physical homogeneity applies) is that the experiences of all experiencers would occur in one space, on top of each other. Each experiencer would be having the experiences of all experiencers (Fig. 10D)!

\subsection{Tension Between Homogeneity and (Typical) Lawfulness, For Multiple Experiencers}

But one space (one coordinate-system!) for all experiences seems counter-factual: we want distinct, individuated, experiences-streams for multiple-experiencers (Fig. 3). Because of apparent problems in making this happen under physically-orthodox homogeneity, for virtual/ontologically-juniorexperiential-space, we ask:

Q6. Lawfulness/Individuated-Multiplicity. 'Does theory-X account for the distinct, individuated, experience-streams of multiple-experiencers - especially if it proposes the same encoding-to-experience associative-laws apply to each experiencer?' 
Figure 10. Multiple Experiencers, Homogeneity, and Individuated Experience-streams. This Figure examines theories-of-experience of the virtual-space kind (Fig. 9D), which propose a sort of 'projection function' $\Pi$ (instantiating experiential-space/contents based on quantification of an experiencer's brain-state). The specific issue here is that statement of $\Pi$ 's algebraic, mathematical, content typically suggests it can be applied indiscriminately to multiple-experiencer populations (Fig. 3A). (This mathematical property is the abstract correlate of physical homogeneity-of-action: 'laws' of physics apply irrespective of location.) A. Stereotypical brain-dynamics $A$ (schematically represented by red-spike) at stereotypical brain-locations $B$ (represented by a red-cross) lead to $\Pi$ projection (purple arrow) of a stereotypical component-of-experience (illustratively depicted by a black square) at a stereotypical location-in-virtual-experiential-space (represented by a red-cross; diagonal dashed-line separates upper-right unseen-realm from lower-left experiential realm [Fig. 1]). B. Because of projection-functions' mathematical structure (and physical correlates of that mathematical structure), dynamics- $A$ at locations- $B$ in a second, distinct, brain lead to the same $E$ at the same $F$. (Of course, the same stimulus should produce the same experience. The point here is that the experientiallocation is not just 'analogous', e.g. as in Fig. 3A: it's identical.) C. To emphasize co-location, the experiences associated with both brains are now depicted simultaneously (with a slight spatial-offset in the square-percept: in fact, two physical brains can't occupy exactly the same spatial point-of-view, so two experiential-representations of a single-stimulus will differ slightly - but they'll still occur in the same, single, experiential-space, according to conventional virtual-experience theories). D. The logical consequence for the total collection of seven-billion-plus human brains is that one experientialspace holds the sum total of all experiences - and so is completely opaque (counter-factually!).
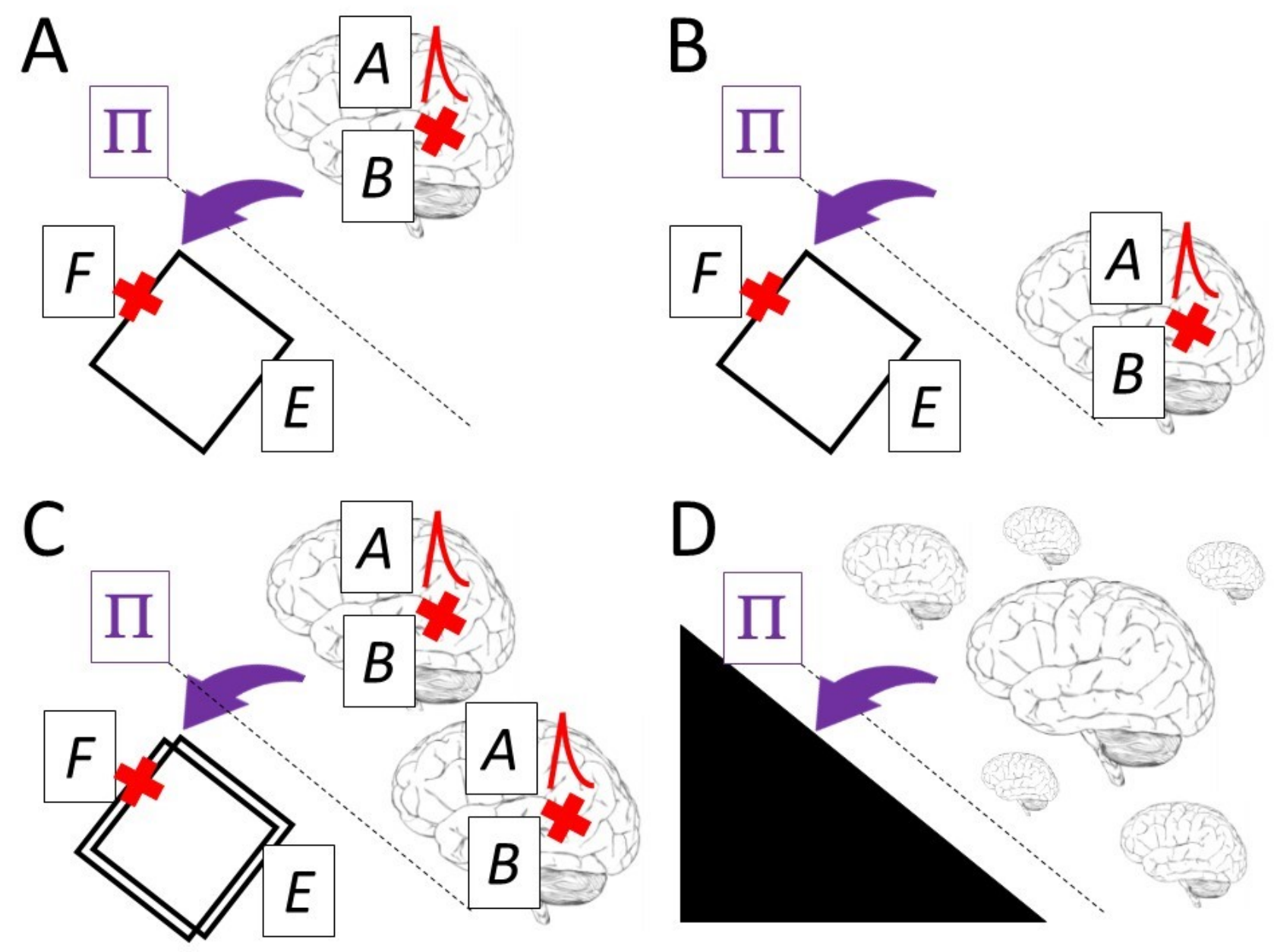


\subsection{Every Mathematically-Complex Theory Suffers Lawfulness/Multiplicity Problems}

The essence of mathematically-complex theories-of-experience is that they propose a formula to apply to numerical measures of brain-dynamical states, which then sets the conditions for association with various kinds of experiences. But those formulae are written in ways that respect homogeneity (presumably in order to explain consciousness in orthodox-physics settings that demand homogeneity). If the formulae were expanded just a little, to include symbolic representations of resulting experientialcombinations, it would become algebraically clear that these theories all suffer the one-space-for-allexperiences problem (subsection 8.4).

Of course, for every algebraic theory, there's an algebraic fix! If an index is added to brain-measures to indicate which experiencer they belong to, an equivalent index can be added to experiential-loci to indicate that these locations relate to that brain. But like most algebraic fixes, this solution has a physical correlate: as discussed in subsection 8.7.1, it simply states that the amended theory-ofexperience violates orthodox physical-homogeneity! Of course, stepping outside orthodox physics is possible - but not when theories are presented as lying inside the orthodox-physics domain (https://academic.oup.com/nc/pages/journal_policies)!

\subsection{Multiple Experiencers and the Unity-of-Consciousness: Potential Solutions}

The multiple-experiencer problem seems more than usually prone to misinterpretation (or denial!) possibly because it is a new formulation (Rosseinsky, 2019), essentially of the unity-of-consciousness problem. (It addresses how experiential-components associated with individual brains can lawfully belong together, as a unity, without having all experiential-components associated with all brains occurring together - if homogeneity holds.) Although the purpose of this paper is really to provide a way to measure field-status against challenges, rather than to suggest solutions, describing a few concrete ways to resolve the lawfulness/individuated-multiplicity problem can help bring the problem itself into focus.

\subsubsection{Solution One: Write Homogeneity Violations into Theories-of-Experience}

Creating multiple/individuated-experience-streams is a problem when neuro-anatomically/neurofunctionally-equivalent locations are treated as homogeneous. Then, salient dynamical-states occurring in any of the homogeneous set should have identical consequences, under homogeneity of action, specifically leading to association with experiential-components at exactly the same experientiallocation.

We could step out of this problem by saying 'brain activity ... in the $i$-th experiencer's brain ... associates with ... experiential-locations in the $i$-th experiential sub-space'. Although solving the onespace-for-all-experience problem, nevertheless this amplifies other extreme problems already faced by mainstream theories, because it suggests Nature does not apply a homogenous physical-law to instantiate experience: instead it must track individual brains as dynamical collections, so it knows which components-of-experience to keep together. Thus, although adding repeated-' $i$-th' content to theories fixes the problem, it does so at the explicit cost of taking theories (further!) outside orthodox physics, because it means they don't follow orthodox homogeneity.

\subsubsection{Solution Two: 'Non-local Long-list Identity'}

Another solution is to write the spatial aspect of a theory-of-experience explicitly in terms of offsets from brain-locations (Fig, 8E). That is, specific activity at $B$-locations leads to experience at an $F$ location that is identical to one of those $B$-locations, or is one of those $B$-locations plus some specified 
vector-offset. This creates individuation by keeping experience 'close' to each brain, and is clearly homogeneous. But in this case, non-locality is explicit, again taking theories outside orthodox physics!

[If we could create a solution of this kind where every $F$-location were identical to a $B$-location, with no non-local information-transfer, we could have homogeneity, individuated multiplicity, and orthodox physics. But we've already excluded this, because it requires a Cartesian Theater.]

\subsubsection{Solution Three: Encoding-of-Experience Varies by Experiencer!}

A third solution would be if the $A$-activity associated with experiential-component- $E$ at experientiallocation- $F$ varied by experiencer. In some arbitrary measure, say $A=1.1$ associates with $\left(E, F_{1}\right)$ for experiencer-1, $A=1.2$ associates with $\left(E, F_{2}\right)$ for experiencer-2, $A=1.3$ associates with $\left(E, F_{3}\right)$ for experiencer-3, and so on. (Here subscript-indices on $F$-symbols indicate equivalent-locations in the experiential-space of the respective experiencer.)

This could be interpreted as a sort of homogeneity, in that $A=1 . \Delta$, at any stereotypical- $B$, leads to an $E$-component at $F_{\Delta}$. And it definitely generates individuated, multiple experience-streams. Unfortunately, it seems impractical. For example, how would Nature coordinate codes-for-experience across multiple-experiencers, to ensure each code was unique?

\section{Q7: Special-Relativistic Consistency}

\subsection{Relativistic Issues Neglected Because of Physics/Neuroscience Split?}

Special-relativistic invariance of theories-of-consciousness is a curious thing: the problem is simultaneously so completely-obvious, and so completely-neglected! Current consciousness science is (or should be!) the confluence of physics and neuroscience. But the current failure of those two disciplines to really meet seems to have obscured relativistic issues for consciousness science.

To physicists, special-relativistic invariance is a must-have (but also allegedly an already-have, because it's built-in to the foundations of physics). Physicists roll their eyes (or laugh out loud) at the idea that there could be something relativistically-interesting about conscious experience, because that must mean that conscious experience lies outside the reduction/emergence realm of current physics. [Which it does (Rosseinsky, 2018b) - but not outside the explanatory reach of every version of physics!]

To neuroscientists, invoking the words 'special relativity' is usually simply bemusing. They were never taught relativity, typically, so they're sure it must be irrelevant. The reason they were never taught it? If biology is a special case of physics (which it is currently, everywhere except in the investigation of consciousness), special-relativistic consistency is built-in to biology (by virtue of being built-in to physics!). So, it seems safe to curriculum-designers to let biologists get $\mathrm{PhDs}$ without fully understanding the limitations and assumptions of the physics-theoretic foundations that biology is built on. (And it is safe - everywhere except in the study of conscious experience ...)

\subsection{Basic Explanation: Relativistic-Invariance-of-Experience Problem for Standard Physics}

It's actually very straightforward to see a relativistic problem for every current theory-ofconsciousness. [For more-detailed accounts, please see (Rosseinsky, 2018b).]

It's widely understood that different relativistic 'observers' (relatively-moving coordinate-systems) can assign different simultaneity-properties to events (Rindler, 1979). Consider the firing of two 
Figure 11. Relativistic Invariance Problems for Typical Theories-of-experience. A. Alice is a human experiencer, and documents events relative to a time-index $t_{\mathrm{A}}$, in the rest-frame of her centerof-gravity. Two neurons in her brain (schematically depicted as circles) are identical to componentsof-experience, when active (depicted as a solid-black fill). Activity of the left-hand neuron is identical to a red-square component-of-experience, right-hand activity to a blue square. (The vertical dashedline separates the schematic depictions of the unseen-realm brain (Fig. 1) and of conscious experience. The same vertical time-coordinate indexes both brain-dynamics and experience. Only three samplesfrom-experience are shown, for graphical clarity.) In $t_{\mathrm{A}}$ terms, the neurons become active at precisely the same time: the first conscious experience containing red or blue squares contains them both. B. For a $B$-labelled coordinate system ('relativistic observer'), moving at a constant velocity relative to Alice, the left-hand neuron become active before the right-hand one. (Simultaneity is now assessed relative to a time-index $t_{\mathrm{B}}$.) $B$-coordinates predict that Alice's first conscious experience containing red or blue squares contains only the red square. $\mathrm{C}$. Another, $C$-labelled, relativistic coordinate-system, has a constant velocity relative to Alice. In $C$-coordinates, the right-hand neuron becomes active before the left-hand neuron. (Simultaneity is now assessed relative to a time-index $t_{\mathrm{C}}$.) $C$-coordinates predict that Alice's first conscious experience containing red or blue squares contains only the blue square. Without explicit amendment to the naïve relativistic treatment of conscious experience, $B$-coordinates (panel $B$ ) and $C$-coordinates (panel $C$ ) both make predictions that conflict with Alice's actual conscious experience (panel $A)$. (B/C-coordinate predictions both contain single-square experiences that are absent from Alice's actual experience-stream, which only ever has both squares together.)
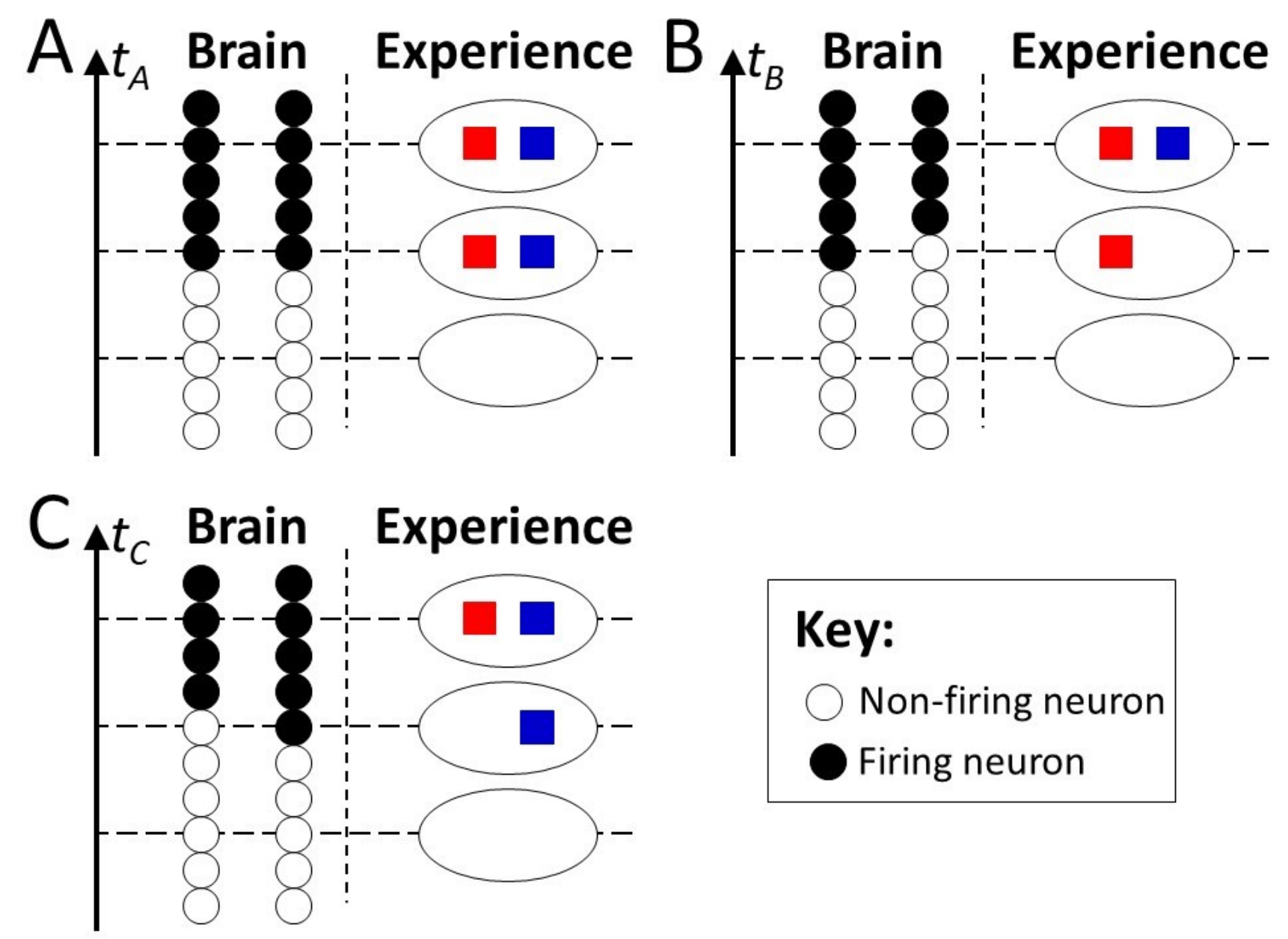

\section{Key:}

Non-firing neuron

Firing neuron 
neurons in Alice's brain that are informationally-significant to experience. Specifically, say that the neuron-one's firing is required for experience-of-red at some experiential-location, and neuron-two's is required for experience-of-blue, somewhere else (Fig. 11). [Although examples are given here in terms of simplistic 'firing-rate' models, emphatically, problems transfer to every current theory-ofconsciousness, including mathematically-complex (Hohwy, 2013; Seth et al., 2011; Tononi et al., 2016) and physically-sophisticated (Hameroff and Penrose, 2014) proposals. The idea (Stapp, 1995) that quantum theories-of-consciousness are automatically relativistically-consistent is also mistaken, as this simple example will show.]

In a coordinate-system $A$, at rest with respect to Alice, say that the two neurons start firing simultaneously. Consequently, experience-of-red and experience-of-blue start together (Fig. 11A). But in another coordinate-system $B$, say that neuron-one starts firing before neuron-two. Then experienceof-red starts before experience-of-blue (Fig. 11B). And in coordinate-system $C$, where neuron-two starts firing before neuron-one, experience-of-red starts after experience-of-blue (Fig. 11C). Thus, Alice's experience is described by observers $B$ and $C$ in ways that differ from her actual experience, assuming that simultaneity-in-experience is the same as neural-simultaneity in her rest-frame. This violates relativistic invariance (natural phenomena must be accurately described in every relativistic coordinate system).

One objection to this analysis says 'we don't know the relationship between simultaneity-in-experience and neural-firing-simultaneity'. But without introducing a specific sort of new physics (which no theory has done, to date), simultaneity-in-experience must correspond to neural-simultaneity in some specific coordinate-system. Say it's coordinate-system X, instead of A. Then B, C and $A$ all wrongly describe actual-experience!

A variation in description of natural-phenomena across relativistic frames is a violation of relativistic invariance. Relativistic invariance is a pre-requisite for every physical theory. So violation-ofrelativistic-invariance is a fundamental problem for theories-of-consciousness. [Variation of simultaneity-in-experience is not the same as variation of simultaneity-of-physical-events. The latter is understood, and well known to be problem-free. The former has not been centrally-acknowledged to date, and lead to problems for current theories-of-consciousness. See (Rosseinsky, 2018b) for a more complete discussion.]

\subsection{The Relativistic Consistency of Theories-of-Consciousness}

As a placeholder for the current relativistic-insufficiency of all theories, we introduce:

Q7: Special-Relativistic Consistency. 'Is theory- $X$ special-relativistically-consistent?'

A variety of suggestions for remedying current relativistic-insufficiencies are discussed in (Rosseinsky, $2018 \mathrm{~b}$ ). These suggestions all require some form of 'new physics'. But as will be explained in subsection 11.3, the Big Five all seem to share a form of 'orthodox-physics imprisonment' mentality, forbidding new physics. Until that changes, relativistic inconsistencies must persist.

\section{Application: The 'Big Five' Theories}

Sections 3 to 9 focused on defining and explaining the seven-question inventory (Table 1). As subsection 2.5 discussed, the question-inventory can be used for value-neutral mapping, or for normative evaluation. This Section and the next illustrate both uses, with reference to the 'Big Five' theories in the current field (Section 1). 


\subsection{Structured Answer-List and Simple Scoring Methodology}

To use the question-inventory to establish a quantified normative-evaluation (subsection 2.5.2.2), in addition to a structured answer-list, we need to establish a scoring-method for specific answers. (For definiteness, more positive numeric-scores indicate greater normative-value.)

\subsubsection{Scoring Q1: Conscious Experience}

Valid Q1-answers are 'yes/perhaps-sometimes/no'. Respective numeric scores are 1/0.5/0. Affirmative answers receive greater credit because of the A1-assumption that theories 'should' study conscious experience, if possible. (However, see also subsection 10.1.6 for the scoring of theories trying to study experience, but in an inconsistent/contradictory setting.)

\subsubsection{Scoring Q2: Causal Efficacy}

Valid Q2-answers are 'yes/partial/no'. Respective numeric scores are 1/0.5/0. Affirmative answers receive greater credit because of the A2-assumption that causal efficacy is required for scientificallymeaningful definitions, discussions, and experiments, concerning experience. (As subsection 10.1.6 discusses, a 'no' answer here with a 'yes' to Q1 therefore establishes inconsistency/contradictoriness.) Additionally, after A5, evolutionary advantage (Q3) also depends on causal efficacy, creating another reason for 'yes'-credit (because evolutionary advantage is also a 'yes'-credit question!).

\subsubsection{Scoring Q3: Direct Evolutionary-Advantage}

Valid Q3-answers are 'yes/no'. Respective numeric scores are 1/0. Affirmative answers receive greater credit because of the A5-assumption that indirect evolutionary explanations currently have infinitesimal probability (e.g. as 'long-list emergence').

\subsubsection{Scoring Q4: Detailed Cortical-Relationships}

Valid Q4-answers are 'yes/partial/no'. Respective numeric scores are 1/0.5/0 Affirmative answers receive greater credit because consciousness science is, in part, about proposing and testing detailed brain/experience-relationships.

\subsubsection{Scoring Q5 to Q7}

In principle, answers for Q5 to Q7 are 'yes/no', with 1/0 scores. However, as discussed in Sections 7 to 9, no current theory addresses these questions satisfactorily, so the Big Five will all be scored at 0. (Theory-by-theory discussions in subsection 10.2 therefore only discuss Q1 to Q4.)

\subsubsection{Should Inconsistent Q1/Q2 Answers Be Scored At 0\% Overall?}

As discussed earlier (Section 1, subsections 2.5.3 and 5.4) and elsewhere (Rosseinsky, 2018a), I take the position that it's explicitly contradictory to claim to be a theory of experience, and simultaneously deny that experience has any causal efficacy. One view is that this contradiction should be reflected in the scoring methodology, for example reducing the overall score to 0 for any theories with Q1'yes'/Q2-'no' combinations. Alternatively, we could introduce a two-factor score, supplementing a sum-total on Q1-to-Q7 with another assessment of scientific-consistency. For this simple introduction, I stick with sum-totals only, in part because Q1/Q2 contradictions are self-evident in the small sample examined.

\subsection{Theory-by-theory evaluation}

Theory-by-theory answers and scores for the seven-question inventory are shown in Table 3. 
Table 3. Three-response Qualitative-Data for the Big Five, and Numeric Scores. Rows depict data for the 'Big Five' theories-of-consciousness ('IIT': integrated information theory; 'GNW': global neuronal workspace; 'HoT': higher-order thought; 'PC': predictive coding; 'OOR: orchestrated objective-reduction). Columns correspond to the seven questions in Table 1 (please refer to Table 1, to understand abbreviated text-descriptions in column headings). 'Answer'/'Score' sub-rows report data from Section 10's analysis, using simple answer-structures (two/three-member lists), for each question. Figure 12 depicts numeric data from the main body of this Table. (Note that Figures 4 and 5, and Table 2 , use an alternative four-answer response-structure, in order to illustrate more sophisticated applications of the same seven-question inventory.) 'Total Score' column reports the simple arithmetic sum of Q1-Q7 row-data. Observe the universally-low total-scores (c. 20\% to 35\%, in 'out-of-seven' terms), and the zero-scores for Q5-to-Q7, for all theories.

\begin{tabular}{|c|c|c|c|c|c|c|c|c|c|}
\hline & & $\begin{array}{c}\text { Q1 } \\
\text { Consc } \\
\text { Exp }\end{array}$ & $\begin{array}{c}\text { Q2 } \\
\text { Causal } \\
\text { Eff }\end{array}$ & $\begin{array}{l}\text { Q3 } \\
\text { Evo } \\
\text { Adv }\end{array}$ & $\begin{array}{c}\text { Q4 } \\
\text { Cort } \\
\text { Det }\end{array}$ & $\begin{array}{c}\text { Q5 } \\
\text { Loc } \\
\text { Geom }\end{array}$ & $\begin{array}{c}\text { Q6 } \\
\text { Lawful } \\
\text { Multi }\end{array}$ & $\begin{array}{l}\text { Q7 } \\
\text { Rel } \\
\text { Inv }\end{array}$ & $\begin{array}{l}\text { Total } \\
\text { Score }\end{array}$ \\
\hline \multirow{2}{*}{ IIT } & Answer & Yes & No & No & Partial & No & No & No & \\
\hline & Score & 1 & 0 & 0 & 0.5 & 0 & 0 & 0 & 1.5 \\
\hline \multirow{2}{*}{ GNW } & Answer & Perhaps & $N o$ & $N o$ & Yes & $N o$ & $N o$ & No & \\
\hline & Score & 0.5 & 0 & 0 & 1 & 0 & 0 & 0 & 1.5 \\
\hline \multirow{2}{*}{ PC } & Answer & Yes & No & No & Partial & No & No & No & \\
\hline & Score & 1 & 0 & 0 & 0.5 & 0 & 0 & 0 & 1.5 \\
\hline \multirow{2}{*}{ HoT } & Answer & Yes & $N o$ & $N o$ & Partial & No & No & No & \\
\hline & Score & 1 & 0 & 0 & 0.5 & 0 & 0 & 0 & 1.5 \\
\hline \multirow{2}{*}{ OOR } & Answer & Yes & Partial & Yes & $\mathrm{No}$ & No & No & $\mathrm{No}$ & \\
\hline & Score & 1 & 0.5 & 1 & 0 & 0 & 0 & 0 & 2.5 \\
\hline
\end{tabular}

\subsubsection{IIT, HoT, PC}

IIT, HoT and PC are examined together as they share the same profile of answers for Q1 to Q4. They are all theories of conscious experience. They all assume a standard-physics setting, which means no causal-efficacy and therefore no (direct) evolutionary-advantage. They are all allocated a 'partial' answer concerning detailed cortical-relationships, because they all contain generic (i.e. not subsection6.5-specific) suggestions of brain-activity/experience relationships (and no suggestions for experienceto-brain coupling - because they deny causal-efficacy).

\subsubsection{GNW}

GNW is treated separately from the $\{$ IIT, HoT, PC $\}$ group. Whether or not GNW is a theory of conscious experience seems to depend on publication, so Q1 is assigned a 'perhaps/sometimes' answer. 
Because GNW originates in a detailed brain-theory, Q4 is answered as 'yes' (which is perhaps generous, given lack of experience-to-brain coupling required for a consistent theory-of-experience; then again, GNW's Q1-answer is 'perhaps/sometimes'!).

\subsection{Orchestrated Objective-Reduction ('Orch-OR')}

Orch-OR is a theory-of-conscious-experience ['collapse creates consciousness' (Hameroff and Penrose, 2014)]. It proposes casual efficacy, e.g. because innovative physical action in Orch-OR is hypothesized to convey mathematical truth, in some circumstances. Nevertheless, it's not clear that the specific kind of causal efficacy proposed in Orch-OR can support detailed, reliable, experiments-aboutexperience. [Orch-OR explicitly proposes transfer-of-information e.g. about mathematical truth, from experience to brain. Reliable experiments require transfer-of-information about detailed contents-ofconsciousness (Rosseinsky, 2018a).] Therefore, the assigned-answer for Q2 is 'partial'. Because enhanced computational capacity proposed by Orch-OR could provide evolutionary advantage [particularly in the explicitly-discussed case of creativity (Penrose, 1989)], Q3 is assigned the (possibly-generous) answer 'yes'. Given the current lack of explicit proposals concerning neuroanatomical/experiential connectivity, Q4 is allocated a 'no' answer.

\subsection{The Big Five: Qualitative and Statistical Summary}

\subsubsection{Statistical Summary}

Table 3 shows 'out-of-seven' sum-totals, for each theory. For the five theories evaluated, the mean percentage-score was $24 \%(1.7 / 7)$, and the median was $21 \%(1.5 / 7)$. The highest scorer was Orch-OR, at $36 \%(2.5 / 7)$.

\subsubsection{Qualitative Summary}

Figure 12 depicts Table 3's numerical results. Features self-evident from verbal analyses so far can be clearly seen. First, all leading mainstream-theories (IIT, GNW, HoT, PC) are deficient in causal and evolutionary aspects. [In one view (subsection 10.1.6), Q1-'yes' with Q2-'no' by itself renders these theories unscientific.] Second, all five theories considered fail to address Q5-to-Q7 concerns at all. Section 11 discusses theoretical terrain neglected by the Big Five, and by the field more generally.

\section{The Neglected Terrain}

\subsection{What's Commonly-Neglected By 'The Big Five'}

\subsubsection{Fixing Q2 Causality Deficits in $\{$ IIT, GNW, HoT, PC}

In a way, it's straightforward to fix causality deficits in what I'm calling the mainstream-theory group (IIT, GNW, HoT, PC). Simply, we can add hypotheses that components-of-experience explained by these theories couple in specific ways with specific report/behavior-related brain-dynamics. In another way, such proposals are antithetical to the apparent philosophical-stance of this group: 'we can explain experience perfectly well, in a standard-physics setting' [(Block et al., 2014); https://academic.oup.com/nc/pages/journal_policies]. Of course, A2 contradicts this stance. It says we can't have any kind of science-of-experience, unless there's causal-efficacy in Nature - which must take us outside standard-physics (Rosseinsky, 2018a).

\subsubsection{Fixing Q4 Cortical-Detail Deficits in Orch-OR}

Similarly, it's straightforward to create explicit Q4-theory-content, coupling specific brainareas/dynamics with novel physical actions proposed in Orch-OR. Here the difficulty might be in 
Figure 12. Simple Graphical Presentation of Scores from Table 3. Rows depict data for the 'Big Five' theories-of-consciousness ('IIT': integrated information theory; 'GNW': global neuronal workspace; 'HoT': higher-order thought; 'PC': predictive coding; 'OOR: orchestrated objectivereduction). Columns correspond to the seven questions in Table 1 (please refer to Table 1 to understand abbreviated text-descriptions in column headings). Colors depict scores ( $\mathrm{red}=0$; orange $=0.5$; green =1) using Section 10's scoring methodology, for its structured-lists of possible answers to the sevenquestion inventory. Note the similar profiles for the four theories in mainstream consciousness science (IIT, GNW, HoT, and PC). (GNW differs from the other three because it is more typically interpreted as an access consciousness theory, rather than a theory-of-experience.) Note also the critical, neglected, theoretical terrain: all five theories discussed here fail to offer any concrete content, for the space/time issues addressed by Q5 to Q7.

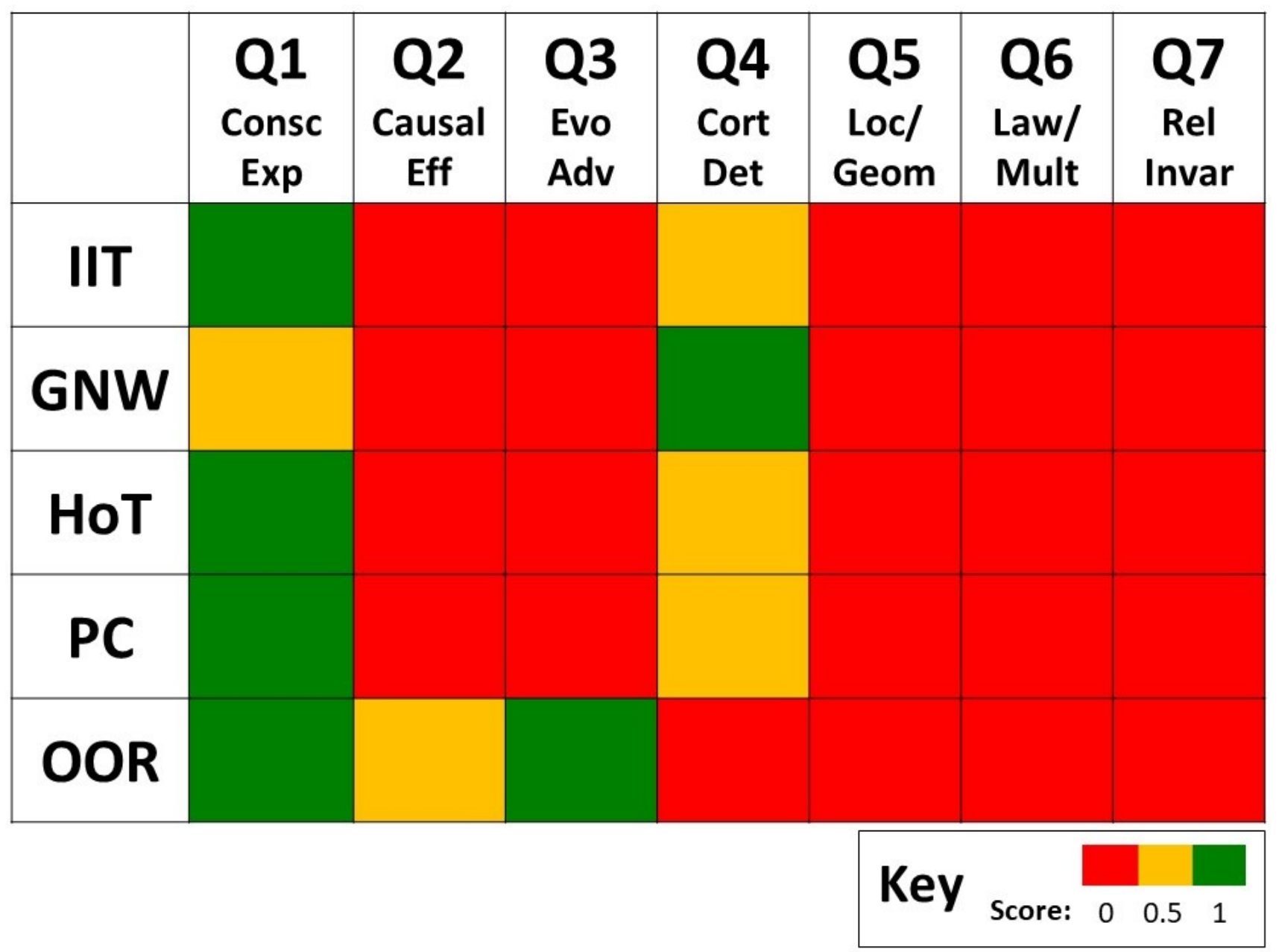

creating a proposal with the right sort of causal-efficacy to explain putative detailed-content coupling. [For example, if 'collapse causes consciousness' (Hameroff and Penrose, 2014), can contents-ofcollapse-caused-consciousness get back into the process-behind-collapse, so that process can then communicate those contents into brain-dynamics, through subsequent collapse?]

\subsubsection{Q5-to-Q7 Are Still Commonly-Neglected After 'Fixes'}

Even if subsections 11.1.1 and 11.1.2 upgrade the Big Five so they're all 100\% on Q1-to-Q4, they'll still all score $0 \%$ on Q5-to-Q7. Of course, it may well be possible to continue to tweak current forms, 
and add new pieces, so that the concerns behind Q5-to-Q7 are addressed. But this kind of work is a major new strand in consciousness science: it's currently being completely neglected.

\subsection{Do Any (Existing) Theories Lie in the Neglected Terrain?}

Of course, it might be that there are existing theories in the field, outside the Big Five, that address Q5to-Q7. Although it is difficult to be certain (given the fragmented nature of the consciousness-related literature), searches in the standard databases did not reveal any such theories.

\subsection{How Could Any (Future) Theory Address the Neglected Terrain?}

One reason to believe that there's no current Q5-to-Q7-satisfactory theory derives from the nature of Q5-to-Q7 problems. These are primarily physics issues, not neuroscience/psychology points, and there's little evidence of physicists as a community engaging with the problem of conscious experience. [Of course, there are notable and welcome individual exceptions (Mukhopadhyay, 2018; Penrose, 1989; Tegmark, 2015).]

Creating satisfactory resolutions for Q5-to-Q7 concerns is by no means impossible (although the challenges should not be underestimated!). I've discussed a range of possible approaches for Q5 and Q7 elsewhere (Rosseinsky, 2018b); some possibilities for Q6 were sketched out in subsections 8.7.2 and 8.7.3. But weaving solutions together, and integrating them with one or more detailed psychobiological theories, e.g. from the Big Five, is quite a project.

One major challenge concerns the 'standard-physics prison' in which the current field seems to want to confine itself. (Even Orch-OR, itself a radically-innovative physical theory, plays this imprisonment game to a degree, in the following sense. Allegedly, the only place to 'put' non-algorithmic computation, given that orthodox-physics 'explains everything else', is in a hypothesis for quantum gravity, as orthodox-physics' last unexplored frontier. Clearly, putative non-algorithmic computation could be hypothetically-associated with something other than quantum-gravity - but that path would suggest there's something other than quantum-gravity that standard-physics doesn't currently explain!)

As I've said at length elsewhere (Rosseinsky, 2018a), Q2-causal-efficacy, sufficient to found a real science of consciousness, must involve non-standard physics, thus taking the mainstream-field outside its current prison. Of course, that step - extending or amending fundamental physics - isn't to be taken lightly. It must be grounded in experimental data (Rosseinsky, 2018a).

The real challenge around Q5-to-Q7, then, is not just coming up with theoretical proposals that could solve the problems. Because Q5-to-Q7 issues again appear to involve fundamental physics (as Q2causality did, in a different way), again we are going to want strong experimental data, to support any further consciousness-science-suggested variations to fundamental constructs. For Q2-causality, at least the experiment's clear [albeit technically demanding (Rosseinsky, 2018a)]. What are the corresponding experiments for arbitrating between various $Q 5-t o-Q 7$ theoretical-options?

\subsection{Dennett's Approach: 0\% Or 100\%?!}

It's often a thankless game to try to reproduce someone else's thought processes. (Even when they explain them!) But here's my attempted-reconstruction of Dennett's 'let's not talk about conscious experience' stance.

If standard physics holds, there's no causal efficacy and no scientifically-meaningful way to talk about experience [A2, (Chalmers, 2018; Dennett, 1991)], or do experiments [A2, (Cohen and Dennett, 2011; 
Rosseinsky, 2018a)]. If orthodox physics holds, we can't solve the locality/recapitulation problem [Section 7, (Dennett and Kinsbourne, 1992)]. No causal-efficacy also means no evolution [A5, Section 5, (Dennett, 1998)].

So, if one assumes orthodox/standard physics really-really-firmly, logically the weight of the evidence is against: a science of experience (no experiments); talking about experience; and even experience's existence (it can't have evolved, and even if it tried to exist, it would violate locality, so it can't exist!).

Thus, Dennett's Q1-to-Q5 stance is actually completely consistent, if we assume orthodox physics is true and unchangeable. (And Q6/Q7 are non-problems for his stance, because experience either doesn't exist, or isn't discussable, or both!)

Let's briefly look at an alternative to the normative-evaluative framework used in Section 10. First, make a fixed assumption of standard physics. (I completely disagree that we are still doing science, after this step. But mainstream consciousness science seems to have adopted it, and should therefore be happy to step into this approach!) Then, simply score theories $0 \% / 100 \%$ on their overall consistency. Dennett's 'we can't talk about experience' then scores 100\%, and, under an A2-view, mainstream theories score $0 \%$ !

My point is that Dennett's stance is the only leading-theory consistent with a rigidly-fixed standardphysics view. Many people seem to think his view is insane (although they'd put it more politely). Certainly, I don't agree with taking standard physics as fixed. But perhaps he doesn't either! Perhaps, he's just reflecting to the mainstream the A2-insanity of presuming standard-physics, and then trying to do conscious-experiential science!

\section{Summary}

The field is currently overlooking a number of major issues, and instead focusing on presentlyirrelevant details.

Firstly, as I've argued elsewhere (Rosseinsky, 2018a), if conscious experience (Section 3) isn't causally-efficacious (Section 4) in a specific way, there are no scientifically-meaningful experiments about consciousness-including-experience, and therefore no science of that-kind-of-consciousness. Above and beyond all other debates, this question must be addressed, first conceptually, and then experimentally. And we need to address the issue together, in the field-as-a-whole.

The causally-efficacious point is also inextricably intertwined with our very capacity to speak about experience (Rosseinsky, 2018a). If there's no causal efficacy, we must be deluding ourselves when talking about experience: experience may be happening, and I may be talking about it, but those must be two parallel and unconnected things. Then, defining experience, talking about it, making theories and so on must all be scientifically-meaningless activities.

Therefore, in the seven-question inventory introduced here (Sections 2 to 7), it's really logicallycontradictory for a theory to claim to be about conscious experience (phenomenal consciousness), and also fail to propose causal efficacy. Yet that is exactly what four out of Section 10's 'Big Five' theories do (and the fifth, Orch-OR, proposes a kind of causal efficacy that isn't definitively sufficient to support defining and discussing conscious experience!). Although many find Dennett's stance puzzling (or even incomprehensible!), at least it seems to be internally-consistent (subsection 11.4): if we assume standard-physics (and so no-causal-efficacy), we can't talk about experience! 
Say we had a set of theories that satisfactorily resolved the efficacy/experience tension. Say these theories also made contrasting experimental predictions, thus in principle offering relative verification/falsifiability. Could we safely test them against each other, to declare an unambiguous theoretical/experimental 'winner' in the consciousness-science stakes? No! There are five further theoretical issues that every candidate must successfully address, before we could say we are anywhere near a coherent explanation of consciousness-including-experience, that's integrated with the rest of science.

Of these five theoretical issues, one - offering direct evolutionary-advantage (Section 5) - can potentially be addressed by just one of the Big Five (again, Orch-OR). However, adding brain/experience-coupling to all the other four - to solve the experience/causality contradiction - could set the stage for further, evolutionarily-complete, sub-versions (subsection 11.1.1). Another requirement - detailed cortical-coupling (Section 6) - is addressed to some degree by four of the five major-theories [but now excluding Orch-OR - although again this deficit could be fixed in a reasonably-straightforward way (subsection 11.1.2)].

However, no major theory currently even mentions the other three issues [locality and the experiential/informational geometry distinction (Section 7), lawful generation of distinct, unified experiences for multiple experiencers (Section 8), and special-relativistic invariance (Section 9)]. And no theory of any kind - major, minor, or imagined - offers any traction on these problems currently. Of course, this doesn't mean the situation is hopeless: I certainly believe that theoretical solutions to these problems will eventually be forthcoming, with the right application of focus and creativity.

But that brings us back to this Section's opening sentence: the field is not currently focusing its creativity on the critical, neglected, theoretical terrain. Thus, although one use of the seven-question inventory is certainly to map and understand the current landscape (Sections 2 and 10, subsection 11.2, and Figs. 4, 5, and 12), the primary takeaway from that exercise should be an exciting call-to-action: there's a tremendous amount missing (Section 11) - which means, a tremendous opportunity!

Again, all of this is necessarily contingent on a point-of-view [e.g. (A1 to A5)]. Without a point-ofview, it's impossible to make any comments - other than to simply list all theories! But is that pointof-view transparent-enough, grounded-enough, inviting-enough, to create a reasoned, respectful, intelligent, co-enquiry, into the truth-of-the-matter, concerning the present state of consciousness science?

\section{Declaration of Contingent Validity}

This paper's discussions of 'phenomenal consciousness' and 'conscious experience' (starting in the second paragraph of Section 1) is only scientifically-valid if Q2's non-standard physics exists in our Universe.

\section{References}

Baars, B.J., 1988. A Cognitive Theory of Consciousness. Cambridge University Press, Cambridge.

Bayne, T., 2018. On the axiomatic foundations of the integrated information theory of consciousness. Neurosci Conscious 2018. https://doi.org/10.1093/nc/niy007

Bayne, T., Spener, M., 2010. Introspective humility. Philosophical Issues 20, 1. 
Berkeley, G., 1878. A treatise concerning the principles of human knowledge. JB Lippincott \& Company, Philadelphia, PA.

Bialek, W., Rieke, F., de Ruyter Van Steveninck, R., Warland, D., 1991. Reading a neural code. Science 252, 1854-1857.

Block, N., 2005. Two neural correlates of consciousness. Trends in cognitive sciences 9, 46-52.

Block, N., Carmel, D., Fleming, S.M., Kentridge, R.W., Koch, C., Lamme, V.A., Lau, H., Rosenthal, D., 2014. Consciousness science: real progress and lingering misconceptions. Trends in cognitive sciences 20,1-2.

Boly, M., Massimini, M., Tsuchiya, N., Postle, B.R., Koch, C., Tononi, G., 2017. Are the neural correlates of consciousness in the front or in the back of the cerebral cortex? Clinical and neuroimaging evidence. J. Neurosci. 37, 9603-9613. https://doi.org/10.1523/JNEUROSCI.3218-16.2017

Bringsjord, S., Xiao, H., 2000. A refutation of Penrose's Gödelian case against artificial intelligence. Journal of Experimental \& Theoretical Artificial Intelligence 12, 307-329.

Chalmers, D.J., 2018. The meta-problem of consciousness. Journal of Consciousness Studies 25, 141.

Chalmers, D.J., 1996. The Conscious Mind: In Search of a Fundamental Theory. Oxford University Press, USA, New York.

Chalmers, D.J., 1995. Minds, machines, and mathematics. Psyche 2, 117-18.

Cleeremans, A., 2003. The unity of consciousness: binding, integration, and dissociation. Oxford University Press, New York.

Cohen, M.A., Dennett, D.C., 2011. Consciousness cannot be separated from function. Trends in Cognitive Sciences 15, 358-364.

Dehaene, S., Changeux, J.-P., 2011. Experimental and theoretical approaches to conscious processing. Neuron 70, 200-227.

Dehaene, S., Lau, H., Kouider, S., 2017. What is consciousness, and could machines have it? Science 358, 486-492. https://doi.org/10.1126/science.aan8871

Dennett, D.C., 1998. The Evolution of Consciousness, in: Toribio, J., Clark, A. (Eds.), Consciousness and Emotion in Cognitive Science: Conceptual and Empirical Issues. Taylor \& Francis, pp. 99-120.

Dennett, D.C., 1991. Consciousness Explained. Little Brown, Boston, MA.

Dennett, D.C., Kinsbourne, M., 1992. Time and the Observer: the Where and When of Consciousness in the Brain. Behavioral and Brain Sciences 15, 183-247.

Desmurget, M., Reilly, K.T., Richard, N., Szathmari, A., Mottolese, C., Sirigu, A., 2009. Movement intention after parietal cortex stimulation in humans. science 324, 811-813.

Earl, B., 2014. The biological function of consciousness. Frontiers in psychology 5, 697.

Elitzur, A.C., 1989. Consciousness and the incompleteness of the physical explanation of behavior. The Journal of Mind and Behavior 10, 1-20.

Ellis, G., 2018. Top-down effects in the brain. Phys Life Rev. https://doi.org/10.1016/j.plrev.2018.05.006 
Fahrenfort, J.J., Lamme, V.A., 2012. A true science of consciousness explains phenomenology: comment on Cohen and Dennett. Trends in cognitive sciences 16, 138-139.

Feldman, J., 2013. The neural binding problem(s). Cognitive Neurodynamics 7, 1-11.

Goff, P., 2017. Can Science Explain Consciousness? Philosophy Now 121, Aug/Sep 2017.

Hameroff, S.R., Penrose, R., 2014. Consciousness in the universe: A review of the 'Orch OR'theory. Physics of life reviews 11,39-78.

Hawking, S.W., 2000. The Objections of an Unashamed Reductionist, in: Longair, M. (Ed.), The Large, the Small and the Human Mind. Cambridge University Press, Cambridge, pp. 169172.

Hohwy, J., 2013. The Predictive Mind. Oxford University Press.

Huxley, T.H., 1893. On the Hypothesis that Animals are Automata, and its History, in: Collected Essays: Volume I, Method and Results. Macmillan, London, pp. 195-250.

Kant, I., Guyer, P., Wood, A.W., 1998. Critique of pure reason. Cambridge University Press.

Klein, S.A., 1995. Is quantum mechanics relevant to understanding consciousness. Psyche 2, 2.

Koch, C., 2018. What Is Consciousness? Nature 557, S8. https://doi.org/10.1038/d41586-018-05097$\mathrm{X}$

Koch, C., Massimini, M., Boly, M., Tononi, G., 2016. Neural correlates of consciousness: progress and problems. Nature Reviews Neuroscience 17, 307-321.

https://doi.org/10.1038/nrn.2016.22

Ladyman, J., 1998. What is structural realism? Studies in History and Philosophy of Science 29, 409-424.

LaRock, E., 2006. Why neural synchrony fails to explain the unity of visual consciousness. Behavior and philosophy 39-58.

Lau, H., Rosenthal, D., 2011. Empirical support for higher-order theories of conscious awareness. Trends in cognitive sciences 15, 365-373.

Lehar, S., 2000. The function of conscious experience: an analogical paradigm of perception and behavior. Consciousness and Cognition 9, 2.

Locke, J., 1998. An Essay Concerning Human Understanding, Revised edition. ed. Penguin Classics, London.

Lucas, J.R., 1961. Minds, Machines and Gödel. Philosophy 36, 112-127.

Maley, C.J., Piccinini, G., 2018. The Biological Evolution of Consciousness, in: Gennaro, R.J. (Ed.), The Routledge Handbook of Consciousness. Routledge.

Melloni, L., Molina, C., Pena, M., Torres, D., Singer, W., Rodriguez, E., 2007. Synchronization of neural activity across cortical areas correlates with conscious perception. The Journal of neuroscience 27, 2858-2865.

Mukhopadhyay, R., 2018. Quantum mechanics, objective reality, and the problem of consciousness. https://arxiv.org/abs/1804.03606

Nagel, T., 1974. What is it like to be a bat? Philosophical Review 435-450. 
Odegaard, B., Knight, R.T., Lau, H., 2017. Should a Few Null Findings Falsify Prefrontal Theories of Conscious Perception? J. Neurosci. 37, 9593-9602.

https://doi.org/10.1523/JNEUROSCI.3217-16.2017

Overgaard, M., 2017. The status and future of consciousness research. Frontiers in psychology 8, 1719.

Paller, K.A., Suzuki, S., 2014. Response to Block et al.: first-person perspectives are both necessary and troublesome for consciousness science. Trends Cogn. Sci. (Regul. Ed.). https://doi.org/10.1016/j.tics.2014.09.008

Parvizi, J., Jacques, C., Foster, B.L., Withoft, N., Rangarajan, V., Weiner, K.S., Grill-Spector, K., 2012. Electrical stimulation of human fusiform face-selective regions distorts face perception. Journal of Neuroscience 32, 14915-14920.

Penrose, R., 1989. The Emperor's New Mind. Oxford University Press, Oxford.

Phillips, I., 2018. The methodological puzzle of phenomenal consciousness. Philosophical Transactions of the Royal Society B: Biological Sciences 373, 20170347. https://doi.org/10.1098/rstb.2017.0347

Quiroga, R.Q., Panzeri, S., 2013. Principles of Neural Coding. CRC Press, Boca Raton, FL.

Rieke, F., de Ruyter van Steveninck, R., Warland, D., Bialek, W., 1997. Spikes: Exploring the Neural Code. MIT Press, Cambridge, MA.

Rindler, W., 1979. Essential Relativity: Special, General, and Cosmological. Springer-Verlag, New York.

Rosseinsky, N.M., 2019. Space - A Final Frontier? Four Space-Related Challenges for the Computational Neuroscience of Consciousness.

Rosseinsky, N.M., 2018a. One Experiment to Start Them All: The Missing Foundation of Consciousness Science. https://doi.org/10.13140/RG.2.2.24360.21765

Rosseinsky, N.M., 2018b. Installing Physical Theory’s Missing Symbolism for Conscious Experience Reveals Ten Puzzles for Science as A Whole. https://doi.org/10.13140/RG.2.2.36652.31361/1

Rosseinsky, N.M., 2015. The measurement of consciousness: assuming completeness of first-person report significantly restricts scope and reliability of theory-discrimination. Front. Psychol. 6. https://doi.org/10.3389/fpsyg.2015.00025

Sahu, S., Ghosh, S., Hirata, K., Fujita, D., Bandyopadhyay, A., 2013. Multi-level memory-switching properties of a single brain microtubule. Applied Physics Letters 102, 123701.

Seth, A.K., 2018a. Consciousness: The last 50 years (and the next). Brain and neuroscience advances $2,1-6$.

Seth, A.K., 2018b. Functions of consciousness. https://doi.org/10.31234/osf.io/wybkp

Seth, A.K., Barrett, A.B., Barnett, L., 2011. Causal density and integrated information as measures of conscious level. Philosophical Transactions of the Royal Society of London A: Mathematical, Physical and Engineering Sciences 369, 3748-3767.

Shoemaker, S., 1982. The inverted spectrum. The Journal of Philosophy 79, 357-381.

Singer, W., 2001. Consciousness and the binding problem. Annals of the New York Academy of Sciences 929, 123-146. 
Smolin, L., 2007. The Trouble with Physics: The Rise of String Theory, The Fall of a Science and What Comes Next. First Mariner, New York.

Smythies, J., 2012. Consciousness and higher dimensions of space. Journal of Consciousness Studies 19, 224-232.

Stapp, H.P., 1995. The integration of mind into physics. Annals of the New York Academy of Sciences 755, 822-833.

Storm, J.F., Boly, M., Casali, A.G., Massimini, M., Olcese, U., Pennartz, C.M., Wilke, M., 2017. Consciousness regained: disentangling mechanisms, brain systems, and behavioral responses. Journal of Neuroscience 37, 10882-10893.

Tegmark, M., 2015. Consciousness as a state of matter. Chaos, Solitons \& Fractals 76, 238-270.

Timmermans, B., Cleeremans, A., 2015. How can we measure awareness? An overview of current methods. Behavioural methods in consciousness research 21-46.

Tononi, G., Boly, M., Massimini, M., Koch, C., 2016. Integrated information theory: from consciousness to its physical substrate. Nature Reviews Neuroscience 17, 450-461. https://doi.org/10.1038/nrn.2016.44

Treisman, A., 2003. Consciousness and perceptual binding. The unity of consciousness: Binding, integration, and dissociation 95-113.

Varela, F., Lachaux, J.-P., Rodriguez, E., Martinerie, J., 2001. The brainweb: phase synchronization and large-scale integration. Nature reviews neuroscience 2, 229-239.

Velmans, M., 2012. The evolution of consciousness. Contemporary Social Science 7, 117-138.

Wagner, M., 2006. The Geometries of Visual Space. Psychology Press, New York.

Wegener, M., 2009. Der psychophysische Parallelismus. NTM Zeitschrift für Geschichte der Wissenschaften, Technik und Medizin 17, 277-316.

Wheeler, J.A., 1990. Information, physics, quantum: The search for links, in: Zurek, W. (Ed.), Complexity, Entropy, and the Physics of Information. Addison-Wesley, Redwood City, CA.

Wilkes, K.V., 1988. ---, yishi, , duh, um, and consciousness, in: Marcel, A., Bisiach, E. (Eds.), Consciousness and Contemporary Science. Clarendon Press, Oxford, pp. 16-41. 